\title{
Modelling dynamics of samples exposed to free-electron-laser radiation with Boltzmann equations.
}

\author{
B. Ziaja ${ }^{* \dagger}{ }^{1}$, A. R. B. de Castro ${ }^{* *}$, E. Weckert ${ }^{*}$ and T. Möller ${ }^{\ddagger}$ \\ * HASYLAB at DESY, Notkestr. 85, D-22603 Hamburg, Germany \\ ${ }^{\dagger}$ Department of Theoretical Physics, Institute of Nuclear Physics, Radzikowskiego 152, \\ 31-342 Cracow, Poland \\ ** IFGW UNICAMP, CEP 13083-970, \\ Campinas, SP Brasil; \\ LNLS, P. O. Box 6192, CEP 13084-971, \\ Campinas, SP Brasil \\ ${ }^{\ddagger}$ Technische Universität Berlin, \\ Institut für Atomare Physik und Fachdidaktik, \\ 10623 Berlin, Hardenbergstrasse 36, Germany
}

\section{January 2006; Revised on 31 July 2006}

PACS Numbers: 41.60.Cr, 52.50.Jm, 52.30.-q, 52.65.-y

\begin{abstract}
We apply Boltzmann equations for modelling the radiation damage in samples irradiated by photons from free electron lasers (FELs). We test this method in a study case of a spherically symmetric xenon cluster irradiated with VUV FEL photons. Qualitative agreement between the model predictions and experimental data is found. The results obtained demonstrate the potential of the Boltzmann method for describing the complex and non-equilibrium dynamics of samples exposed to FEL radiation.
\end{abstract}

\footnotetext{
${ }^{1}$ Author to whom correspondence should be addressed

2Electronic mails: ziaja@mail.desy.de, arbcastro@lnls.br, edgar.weckert@desy.de, thomas.moeller@physik.tu-berlin.de
} 


\section{Introduction}

The emerging free-electron-lasers (FELs) promise tremendous progress in studying the structure of matter with soft and hard X-rays. The transversely fully coherent radiation from the FEL will be delivered in flashes of ultrashort duration, emitted at peak brilliances more than $10^{8}$ higher than those available from the present sources of synchrotron radiation $[1,2]$. These unique properties of FELs enable probing dynamic states of matter, transitions and reactions happening within tens of femtoseconds, with wide-ranging implications to the solid state physics, material sciences, and to the femtochemistry. The FEL beam, if focussed onto a very small spot, is also an excellent tool to generate and probe extreme states of matter. The X-ray FEL (XFEL) is expected to open new horizons in structural studies of biological systems, especially in the studies of non-repetitive samples, like viruses, living cells etc. Rapid progress of radiation damage in these samples prevents an accurate determination of their structure in standard diffraction experiments. However, the recent studies of the progress of the damage formation [3-5] indicate that the radiation tolerance might be extended at ultrafast imaging with high radiation dose as that expected with the presently developed X-ray FELs (LCLS, DESY).

For this and other applications of FELs, we have to understand in detail how the intense radiation of short wavelengths, emitted in short pulses, interacts with matter. In particular, accurate time characteristics of radiation damage is necessary in order to estimate the range of pulse length at which imaging with XFEL would be possible.

The radiation damage of samples irradiated by soft X-rays differs considerably from that induced by high power IR-Lasers. In the latter case plasma heating by inverse bremsstrahlung is the dominant damage process [6-8]. For FELs, at different energies of the FEL photons different processes are contributing to the radiation damage. At VUV photon energies the inverse bremsstrahlung process is believed to deliver most of the energy needed for the efficient ionization of an irradiated system [9]. This ionization eventually leads to the Coulomb explosion of the sample. Photons of shorter wavelengths may excite electrons from inner shells of atoms, creating core holes [10]. Photoemissions, core-hole creations and subsequent Auger emissions of secondary electrons contribute to the radiation damage that then affects not only the sample but also the optical elements of the FEL beamline.

Radiation damage by photons from the VUV FEL is now under intense investigation. First experimental studies on the interaction of the VUV photon beams with atoms, molecules and clusters have been already performed at DESY [11]. The large number of VUV photons absorbed per atom that was observed in these experiments could not be explained using the well-established standard calculations for photon absorption $[9,11]$. This indicated that the 
ionization of samples irradiated by energetic photons progresses in a different way than that observed in the optical energy range. These surprising results stimulated intense theoretical effort. Several interesting models have been proposed [9, 12-14] which could explain various aspects of the increased photoabsorption and ionization dynamics observed in the experiments (for review see [15]). On the other hand, there are still some controversies, e. g. regarding the role of the inverse bremsstrahlung mechanism and the inner ionization processes. A model: (i) computationally efficient also for large spatially non-uniform samples and (ii) able to test the influence of specific interactions on the complex dynamics of electrons and ions, could be useful in evaluating the contributions of different mechanisms to the overall ionization dynamics.

Here we propose such efficient method of describing the evolution of irradiated samples which applies also to large systems. This first-principles Boltzmann method is based on the statistical description of the charge dynamics in terms of statistical quantities: electron and ion densities in phase space, $\rho^{(e, i)}(\mathbf{r}, \mathbf{v}, t)$. These densities are functions of the spatial and velocity coordinates, $\mathbf{r}$ and $\mathbf{v}$, and are measured at some time, $t$. The quantity, $\rho^{(e, i)}(\mathbf{r}, \mathbf{v}, t) d^{3} r d^{3} v$, estimates a number of particles (electrons or ions) in an infinitesimal volume element of phase space, $d V=d^{3} r d^{3} v$, which is located at the spatial point, $\mathbf{r}$, and at the velocity, v. Charge densities are evolved from their initial configuration at $t=0$, using semiclassical Boltzmann equations.

The Boltzmann method is a promising alternative to the first-principles Monte Carlo (MC) or Molecular Dynamics (MD) methods which are commonly used [3,4,16]. The Monte Carlo method may be viewed as an approximate stochastic method of solving transport equations. During the simulation the MC code has to solve the separate equations of motion for each particle in the sample, following the trajectories of all particles and their interactions with other particles or external fields. If any scatterings occur, the scattering probabilities are estimated with quantum mechanical cross sections. Coordinates and velocities of particles are updated at each time-step. Simulations of single events are repeated many times. Estimates of physical observables are obtained by averaging their values obtained from single events over the total number of events. Therefore these estimates are biased with statistical errors.

Monte Carlo algorithms have a transparent structure and usually do not require an application of any complex numerical methods. This is a great advantage of this method. However, these algorithms become computationally inefficient when the number of particles, $N$, is large. The code efficiency is even worse, if the long-range interactions between particles (e.g. Coulomb electrostatic forces) have to be included into the simulation. High computational costs which scale with the number of particles restrict the applicability of the 
Monte Carlo method to the samples of small or moderate sizes.

In contrast, the efficiency of the simulation algorithm with the Boltzmann equations does not change directly with the number of particles in the sample, as the algorithm operates on smooth density functions. Therefore the efficiency and accuracy of these algorithms depend only on the phase-space shape of the sample which is reflected in the number of grid points used in the simulation. Therefore this statistical approach can also work fine for large samples, where the MD/MC methods are inefficient.

The full spatio-temporal characteristics of the electron and ion dynamics can be easily obtained with the transport method. As charge densities are directly evolved with Boltzmann equations, the averaged observables, $O$, of interest can then be calculated with their convolution with the charge densities obtained, $\langle O(t)\rangle=\int O(\mathbf{r}, \mathbf{v}) \rho(\mathbf{r}, \mathbf{v}, t) d^{3} r d^{3} v$. These results are not biased with statistical errors.

The applicability of Boltzmann equations is, however, limited to the systems which fulfill the assumptions of molecular chaos and two-body collisions. These assumptions are usually justified by a presence of short range forces $[17,18]$. The single particle density function obtained with these equations does not contain any information on three-body and higher correlations. If the higher order correlations are important, a more fundamental Liouville equation for the N-particle density function should be applied. The Liouville equation reduces to the collisionless Vlasov equation [17] in case of an uncorrelated system. Fokker-Planck equation [17] can be derived as a limiting form of the Liouville equation for long-range (Coulomb) forces. It was proven in Ref. [17] that a correct description of many body long-range interactions of plasma electrons and ions obtained with the dedicated Fokker-Planck equations can be also obtained with the two-body Boltzmann collision term, assuming the Debye cutoff in the Rutherford scattering cross section. This simplification does not apply to the electronelectron interactions, where the interacting charged particles have identical masses, and the momentum transfer during their collisions cannot be neglected.

Another disadvantage of the Boltzmann approach is its numerical complexity. Boltzmann equations are complicated sets of nonlinear integro-differential equations where partial derivatives appear in both spatial and velocity coordinates, $\partial / \partial \mathbf{r}$, and $\partial / \partial \mathbf{v}$. Advanced numerical methods have then to be applied.

In what follows we will show the potential of Boltzmann method for studies of the radiation damage in samples irradiated by FEL photons. At the present state we do not aim to obtain any quantitative predictions which could later be compared to the existing experimental data. Actually, we are interested in proposing a new theoretical approach for a comprehensive description of the progress of radiation damage in irradiated samples, almost 
independently of the sample size. A construction of a realistic model including all relevant physical processes is planned at later stages.

First we will write general Boltzmann equations for samples irradiated with VUV photons. These equations will include basic physical processes contributing at those photon energies. We will then solve these equations in a simplified (study) case of a spherically symmetric xenon cluster. Due to the symmetries of the sample which eliminate the dependence of charge densities on one of the azimuthal angles, the number of independent coordinates in these equations can be reduced by one, from six to five. Further simplification of Boltzmann equations is achieved by applying the first-order angular moments expansion to the charge densities. These simplified equations, containing three different components of charge density: the isotropic, transport and the drift one, can then be treated numerically in an efficient way.

We will solve these simplified Boltzmann equations, and show how efficiently they can follow the dynamics of electrons. We will consider two study cases: (i) the case of the pure Coulomb dynamics, where we restrict the interaction of the laser field with the sample only to the photoionization effect, (ii) the case where a complete interaction of sample with the laser field is implemented, i.e. the drift component of electron density describing the interaction of electron density with laser field is treated, and the contribution of the inverse bremsstrahlung process which heats up electrons during elastic electron-ion collisions is included. The results obtained are discussed in detail. Afterwards, a short summary is given. Finally, we list our conclusions.

\section{Boltzmann equation}

Statistical description of a classical system can be made in terms of its density function, $\rho(\mathbf{r}, \mathbf{v}, t)$ [18-20], where $\rho(\mathbf{r}, \mathbf{v}, t)$ is defined such that $\rho(\mathbf{r}, \mathbf{v}, t) d^{3} r d^{3} v$ is the number of particles at time $\mathbf{t}$ positioned between $\mathbf{r}$ and $\mathbf{r}+\mathbf{d r}$ which have velocities in the range $(\mathbf{v}, \mathbf{v}+\mathbf{d} \mathbf{v})$.

Evolution of this density function can then be described by Boltzmann equation,

$$
\partial_{t} \rho+\mathbf{v} \partial_{\mathbf{r}} \rho+\mathbf{F} \partial_{\mathbf{v}} \rho / m=\Omega(\rho, \mathbf{r}, \mathbf{v}, t)
$$

where $F$ is a force (external of internal) acting within the system, and $\Omega(\rho, \mathbf{r}, \mathbf{v}, t)$ is a collision (source) operator, describing the change of charge density due to the interparticle collisions or other short range processes ocurring within the system.

The most important feature of Boltzmann equation is the ability to describe non-equilibrium 
processes. Boltzmann equations are used for describing transport phenomena in many different physical contexts, ranging from the simulations of the hot electron transport in semiconductors, simulations of plasma kinetics [17,21-24] to the evolution of protoneutron stars [25] and to the modelling of the core collapse in supernovas [26].

We will now formulate the specific Boltzmann equations describing the transport of electrons, atoms and ions inside a sample irradiated with FEL photons. In this case it is enough to consider two gases: the gas of light electrons of masses, $m$ and charges, $-e$, and the gas of heavy atoms/ions of masses, $M$, and charges, $i e$. Photons need not to be considered as an independent gas component, as they only enter the equations as a flux term in the photoionization source term. The gases of electrons and atoms/ions are represented by the density functions: $\rho^{(e)}(\mathbf{r}, \mathbf{v}, t), \rho^{(i)}(\mathbf{r}, \mathbf{v}, t)$, where $i$ denotes the ion charge $i=0,1, \ldots, N_{J}$, and $N_{J}$ is an arbitrary number, describing the maximal ion charge in the system. The general coupled Boltzmann equations for these gases are:

$$
\partial_{t} \rho^{(e)}(\mathbf{r}, \mathbf{v}, t)+\mathbf{v} \cdot \partial_{\mathbf{r}} \rho^{(e)}(\mathbf{r}, \mathbf{v}, t)+\frac{e}{m}(\mathbf{E}(\mathbf{r}, t)+\mathbf{v} \times \mathbf{B}(\mathbf{r}, t)) \cdot \partial_{\mathbf{v}} \rho^{(e)}(\mathbf{r}, \mathbf{v}, t)=\Omega^{(e)}\left(\rho^{(e)}, \rho^{(i)}, \mathbf{r}, \mathbf{v}, t\right),
$$

for electrons, and

$$
\partial_{t} \rho^{(i)}(\mathbf{r}, \mathbf{v}, t)+\mathbf{v} \cdot \partial_{\mathbf{r}} \rho^{(i)}(\mathbf{r}, \mathbf{v}, t)-\frac{i e}{M}(\mathbf{E}(\mathbf{r}, t)+\mathbf{v} \times \mathbf{B}(\mathbf{r}, t)) \cdot \partial_{\mathbf{v}} \rho^{(i)}(\mathbf{r}, \mathbf{v}, t)=\Omega^{(i)}\left(\rho^{(e)}, \rho^{(i)}, \mathbf{r}, \mathbf{v}, t\right),
$$

for atoms/ions, where the force $\mathbf{F}$ is the electromagnetic force, $\mathbf{F}(\mathbf{r}, \mathbf{v}, t)=q(\mathbf{E}(\mathbf{r}, t)+\mathbf{v} \times$ $\mathbf{B}(\mathbf{r}, t))$, acting on electrons and ions positioned between $\mathbf{r}$ and $\mathbf{r}+\mathbf{d r}$, which have velocities in the range $(\mathbf{v}, \mathbf{v}+\mathbf{d v})$. The electric field, $\mathbf{E}$, and magnetic field, $\mathbf{B}$, have two components. The first component describes the interaction of charges with external radiation. The second component describes internal electromagnetic interaction between electrons and ions. This component is a non-local function of electron and ion densities.

Collision terms, $\Omega^{(e, i)}$, describe the change of the electron/ion densities of velocities $(\mathbf{v}, \mathbf{v}+\mathbf{d v})$ measured at the positions $(\mathbf{r}, \mathbf{r}+\mathbf{d r})$ with time. This change may be due to: (i) the creation of the secondary electrons and highly charged ions via photo- and collisional ionizations of atoms and ions, (ii) elastic and inelastic collisions of electrons and ions, (iii) the inverse bremsstrahlung process, i. e. absorptions and emissions of photons by electrons during the elastic electron-ion collisions, (iv) recombination processes etc. Number of shortrange processes involved in the sample dynamics depends on the wavelength of the laser radiation. If collision terms are neglected, Boltzmann equations, Eqs. (2), (3), reduce to the Vlasov equation $[17,21]$ describing the evolution of a collisionless plasma.

Initial configuration of Eqs. (2), (3) is given by a smooth atomic density function, $\rho^{(0)}(\mathbf{r}, \mathbf{v}, 0)$, which represents the sample at $t=0$. 


\section{Boltzmann equation for an irradiated atomic cluster}

First experimental studies on the interaction of intense VUV photon beams with matter were performed for clusters of xenon atoms irradiated with VUV photons [11,27]. New experiments with clusters exposed to FEL radiation at higher photon energies are planned in the next future. The existing and the future experimental data give a unique opportunity for testing theoretical models.

Below we formulate the assumptions of the primary transport model dedicated for studying the dissipative dynamics and the radiation damage in xenon clusters at the VUV photon energies. We will fix the physical parameters as they were set in the first experiment with the VUV photons [11].

The production terms, $\Omega^{(e, i)}$, in our model will then include only basic predominant interactions, i.e.:

(i) Single photoionizations of atoms. A single VUV photon of energy, $E_{\gamma}=12.7$ $\mathrm{eV}$, may excite electrons only from the $5 p_{3 / 2}$ shell of xenon atoms of the binding energy, $E_{i}=12.1 \mathrm{eV}$. Here the photon energy was set as in the VUV FEL experiment [11]. We neglect possible multistep photo- and multiphoton ionizations within this primary model. We also neglect the effect of plasma screening on the atomic energy levels and photoionization cross sections.

(ii) Elastic and inelastic collisions of electrons and atoms/ions. We assume that an inelastic collision always releases a secondary electron. We neglect inelastic collisions of electrons and atoms/ions which lead only to an excitation of an atom/ion. These processes contribute to the multistep collisional ionization which is not included within this primary model. We also neglect the effect of plasma screening on the collisional cross sections.

(iii) Inverse bremsstrahlung photoabsorption in the presence of atoms or ions. In our model, as the primary kinetic energy of a photoelectron released by a VUV photon is small, $E \sim 0.6 \mathrm{eV}$, comparing to the first ionization energy, $E_{i}=12.1 \mathrm{eV}$, a process of energy pumping is necessary in order to initiate any collisional ionizations by electrons. Inverse bremsstrahlung process is among the possible processes [9]. At the low photoelectron energies, that we consider here, the proper description of inverse bremsstrahlung should be quantum and not classical [28,29]. Quantum cross sections for absorption or emission of radiation photons by electrons during their collisions with ions were taken from Ref. [30]. In this approximation ions were treated as point-like charges [30].

(iv) Electromagnetic interaction of electrons with laser field. Here this interaction is treated within the dipole approximation. This approach is justified by the small spatial size 
of the irradiated spherical cluster of a radius $\sim 25 \AA$, when compared to the wavelength of laser radiation $(\sim 100 \mathrm{~nm})$. We expect that the attenuation of the laser beam is small. Rough calculation of the attenuation via photoabsorption at the beginning of the pulse gives the transmission of about $94 \%$. After all atoms have been photoionized, photons from the pulse can be still absorbed via inverse bremsstrahlung. Estimated total energy absorbed by this xenon cluster is about 300 thousand of eVs at most.

(v) Electromagnetic interactions of electrons and ions within the sample. They are expressed in the form of the non-local potentials. We assume for simplicity that both electrons and ions are point-like charged particles, and neglect the effects of the atom/ion internal structure and of its finite size on the interaction potential within this primary model.

Finally, we note that within this primary model we also neglect the recoil energies and the recoil momenta of the atoms/ions gained during their interactions with photons or electrons. Electrons are assumed to scatter isotropically on atoms/ions. This is the first order approximation which can be made: (i) in case of photoionizations due to the low energy of the incoming photons, and (ii) in case of collisional interactions due to the large difference of electron and ion masses and to the low impact energies of electrons. Within this approximation the movement of ions will be stimulated by the Coulomb repulsion only, and will start at the final stages of the explosion. Additional pressure on ions due to the recoil momenta is neglected.

Recoil effects, and also short-range electron-electron interaction can be conveniently treated by the means of the Fokker-Planck equation. As other relevant processes which were neglected within this primary model, e.g. three-body recombination, charge enhanced ionization [12] or effects of electron screening [9], these processes will be treated in forthcoming papers.

\subsection{General Boltzmann equations for electrons and ions in an irradi- ated cluster}

Before writing the equations, we will introduce the following notation. The integrated densities, $\bar{\rho}^{(e, i)}(\mathbf{r}, t)$ are defined as,

$$
\bar{\rho}^{(e, i)}(\mathbf{r}, t)=\int d^{3} v \rho^{(e, i)}(\mathbf{r}, \mathbf{v}, t) .
$$

Velocity $v_{E}=\sqrt{2\left(E_{\gamma}-E_{i}\right) / m}$ is the magnitude of the velocity of the photoelectrons. Co-

efficients, $\sigma_{\gamma}^{i \rightarrow i+1}$, denote the total photoionization cross sections for a single ionization of an ion of charge, $i=0,1, \ldots, N_{J}$. Ionizations up to $N_{J}=7$ are allowed within our model. 
Coefficients, $\sigma_{i c}^{i \rightarrow i+1}$, denote the total collisional cross sections for a single ionization of ions of charge, $i=0,1, \ldots, N_{J}$ by an electron. Coefficients, $\sigma_{e c}^{i \rightarrow i}$, denote the elastic collisional cross sections. Elastic collisional cross sections and ionization collisional cross sections were measured experimentally for xenon [31-35]. Within this primary model the cross section for the elastic electron-ion scattering was approximated by the cross section for the elastic electron-atom scattering. The scattering of electrons on ions was assumed to be isotropic.

A compact notation for doubly differential cross sections is used, $\frac{d \sigma^{i \rightarrow j}\left(\boldsymbol{v}_{\boldsymbol{e}} ; \boldsymbol{v}_{\boldsymbol{e}}^{\prime}\left(\boldsymbol{v}_{\boldsymbol{s}}\right)\right)}{d \mathbf{v}}$. Velocity $\mathbf{v}_{e}$ denotes the velocity of the incoming electron, $\mathbf{v}_{e}^{\prime}$ is the velocity of this electron after a collision, $\mathbf{v}_{s}$ is the velocity of the secondary electron.

Coefficient $j\left(E_{\gamma}\right)$ describes the photon flux, and $\Omega$ is a spherical angle.

Starting from Eqs. (2), (3), we derive the following equations for electron and ion densities within an irradiated sample:

$$
\begin{aligned}
& \frac{\partial \rho^{(e)}(\mathbf{r}, \mathbf{v}, t)}{\partial t}+\mathbf{v} \frac{\partial \rho^{(e)}(\mathbf{r}, \mathbf{v}, t)}{\partial \mathbf{r}}- \\
&- \frac{\partial \rho^{(e)}(\mathbf{r}, \mathbf{v}, t)}{\partial \mathbf{v}} \cdot\left(\frac{e^{2}}{4 \pi \epsilon_{0} m} \int d^{3} r^{\prime} \frac{\mathbf{r}-\mathbf{r}^{\prime}}{\left|\mathbf{r}-\mathbf{r}^{\prime}\right|^{3}}\left\{\sum_{i=0}^{N_{J}} i \cdot \bar{\rho}^{(i)}\left(\mathbf{r}^{\prime}, t\right)-\bar{\rho}^{(e)}\left(\mathbf{r}^{\prime}, t\right)\right\}+\frac{e}{m} E(t) \boldsymbol{\epsilon}\right)= \\
&= \sum_{i=0}^{N_{J}} \bar{\rho}^{(i)}(\mathbf{r}, t) j\left(E_{\gamma}\right) \frac{d \sigma_{\gamma}^{i \rightarrow i+1}\left(E_{\gamma} ; \mathbf{v}\right)}{d \Omega_{\mathbf{v}}} \frac{\delta\left(v-v_{E}\right)}{v^{2}}+ \\
&+ \sum_{i=0}^{N_{J}} \bar{\rho}^{(i)}(\mathbf{r}, t)\left\{\int d^{3} v_{e} v_{e} \rho^{(e)}\left(\mathbf{r}, \mathbf{v}_{\mathbf{e}}, t\right) \frac{d \sigma_{e c}^{i \rightarrow i}\left(\mathbf{v}_{\mathbf{e}} ; \mathbf{v}\right)}{d \Omega_{e v}} \frac{\delta\left(v-v_{e}\right)}{v^{2}}-v \rho^{(e)}(\mathbf{r}, \mathbf{v}, t) \sigma_{e c}^{i \rightarrow i}(\mathbf{v})\right\}+ \\
&+ \sum_{i=0}^{N_{J}} \bar{\rho}^{(i)}(\mathbf{r}, t)\left\{\int d^{3} v_{e} v_{e} \rho^{(e)}\left(\mathbf{r}, \mathbf{v}_{\mathbf{e}}, t\right)\left(\frac{d \sigma_{i c}^{i \rightarrow i+1}\left(\mathbf{v}_{\mathbf{e}} ; \mathbf{v}_{\mathbf{e}}^{\prime}=\mathbf{v}\right)}{d \mathbf{v}}+\frac{d \sigma_{i c}^{i \rightarrow i+1}\left(\mathbf{v}_{\mathbf{e}} ; \mathbf{v}_{\mathbf{s}}=\mathbf{v}\right)}{d \mathbf{v}}\right)-\right. \\
&+\left.-v \rho^{(e)}(\mathbf{r}, \mathbf{v}, t) \sigma_{i c}^{i \rightarrow i+1}(\mathbf{v})\right\} \\
& \sum_{i=1}^{N_{J}} \bar{\rho}^{(i)}(\mathbf{r}, t)\left\{\sum_{n=-\infty}^{\infty} \int d^{3} v_{e} d^{3} v_{e}^{\prime} \rho^{(e)}\left(\mathbf{r}, \mathbf{v}_{\mathbf{e}}, t\right)\left[\delta\left(\mathbf{v}_{\mathbf{e}}^{\prime}-\mathbf{v}\right)-\delta\left(\mathbf{v}-\mathbf{v}_{\mathbf{e}}\right)\right]\right. \\
& \times \delta\left(\frac{v_{e}^{\prime 2}}{2}-\frac{v_{e}^{2}}{2}-\frac{n \hbar \omega}{m}\right) J_{n}^{2}\left(-\frac{e E_{0}}{\hbar \omega^{2}} \boldsymbol{\epsilon}\left(\mathbf{v}_{\mathbf{e}}^{\prime}-\mathbf{v}_{\mathbf{e}}\right)\right) \frac{d \sigma_{e c}^{i \rightarrow i}\left(\mathbf{v}_{\mathbf{e}}^{\prime}-\mathbf{v}_{\mathbf{e}}\right)}{\left.d \Omega_{e l}\right\}}
\end{aligned}
$$

for electrons and:

$$
\begin{aligned}
& \frac{\partial \rho^{(i)}(\mathbf{r}, \mathbf{v}, t)}{\partial t}+\mathbf{v} \frac{\partial \rho^{(i)}(\mathbf{r}, \mathbf{v}, t)}{\partial \mathbf{r}}+ \\
+ & \frac{\partial \rho^{(i)}(\mathbf{r}, \mathbf{v}, t)}{\partial \mathbf{v}} \cdot\left(\frac{e^{2}}{4 \pi \epsilon_{0} M} \int d^{3} r^{\prime} \frac{\mathbf{r}-\mathbf{r}^{\prime}}{\left|\mathbf{r}-\mathbf{r}^{\prime}\right|^{3}}\left\{\sum_{j=0}^{N_{J}}(i j) \cdot \bar{\rho}^{(j)}\left(\mathbf{r}^{\prime}, t\right)-i \bar{\rho}^{(e)}\left(\mathbf{r}^{\prime}, t\right)\right\}+\frac{i e}{M} E(t) \boldsymbol{\epsilon}\right)= \\
= & j\left(E_{\gamma}\right) \sigma_{\gamma}^{i-1 \rightarrow i}\left(E_{\gamma}\right) \rho^{(i-1)}(\mathbf{r}, \mathbf{v}, t)-j\left(E_{\gamma}\right) \sigma_{\gamma}^{i \rightarrow i+1}\left(E_{\gamma}\right) \rho^{(i)}(\mathbf{r}, \mathbf{v}, t)+ \\
+ & \left\{\int d^{3} v_{e} \sigma_{i c}^{i-1 \rightarrow i}\left(\mathbf{v}_{\mathbf{e}}\right) v_{e} \rho^{(e)}\left(\mathbf{r}, \mathbf{v}_{\mathbf{e}}, t\right)\right\} \rho^{(i-1)}(\mathbf{r}, \mathbf{v}, t)-
\end{aligned}
$$




$$
-\left\{\int d^{3} v_{e} \sigma_{i c}^{i \rightarrow i+1}\left(\mathbf{v}_{\mathbf{e}}\right) v_{e} \rho^{(e)}\left(\mathbf{r}, \mathbf{v}_{\mathbf{e}}, t\right)\right\} \rho^{(i)}(\mathbf{r}, \mathbf{v}, t)
$$

for ions, where we treated the interaction of charges with the external electric field ( also inverse bremsstrahlung for electrons) within the dipole approximation,

$$
\mathbf{E}(\mathbf{r}, t) \cong E(t) \boldsymbol{\epsilon}
$$

and neglected the subleading contribution coming from the interaction of charges with magnetic field. Vector, $\boldsymbol{\epsilon}$, is the polarization vector of the electric field.

Writing these equations we took into account only binary collisions between participating particles. The assumption of binary collisions is not valid for very dense systems and for systems with the presence of long-range forces, where many body effects become important [14]. Within this primary model we neglected short-range three and higher order many body interactions ocurring due to the high density of particles in the sample. Many body effects due to the presence of Coulomb forces were treated correctly within the approximation that recoil energies and recoil momenta of atoms and ions could be neglected.

Equations (5), (6) then describe the evolution of a cluster irradiated with VUV FEL photons within our primary model. The structure of these equations is general, and other interactions or improvements can conveniently be implemented into these equations. These completed equations would then describe a more advanced model of the sample dynamics. Eqs. (5), (6) can be also adapted for describing the dynamics of an irradiated sample at other photon energies.

\subsection{Solving the Boltzmann equations}

Equations (5), (6) are complicated integro-differential equations in six-dimensional phase space. They can be treated only numerically. For a spherically symmetric cluster the number of dimensions can be reduced by one, from six to five. A significant simplification of the Boltzmann equations (5), (6) can be achieved by expanding the electron and ion densities in terms of their angular moments. This method was successfully applied for the description of the evolution of the protoneutron stars [25] and plasmas [17,21]. An assumption has then to be made that the isotropic components of the electron and ion densities are predominant. Here we mean isotropy in phase space, and not only in space, i.e. at each spatial point of an isotropic spatial distribution the velocity distribution has also to be isotropic. Such approximate isotropy occurs in systems where there is a strong collisional dissipation of particle energies, and the phase space component of the collective transport is small. This is certainly the case for low energy electrons inside an ionic/atomic cluster or gas, as they then frequently collide (with short range forces) with ions and atoms inside this sample. 
The validity of the angular moments method may be also verified a posteriori, i.e. after solving the Boltzmann equations one may compare the magnitude of the isotropic component of the charge densities to the angular ones. If the isotropic components of the electron and ion densities obtained with these equations were much larger that the other components of the electron densities, the angular moments approximation worked correctly, and the solution of the equation obtained within this approximation is a good estimate of the full solution.

\section{Test of Boltzmann method: simplified electron dynamics inside an atomic cluster}

In order to test the applicability and efficiency of the Boltzmann method for describing the dynamics of electrons within FEL irradiated samples, we have applied it to two study cases of a simplified electron dynamics: (i) the case of pure Coulomb dynamics, where the interaction of sample with the laser field was restricted to photoionization effect, (ii) the case where the complete interaction of the sample with laser field was included, i.e. the drift component of the electron density describing the interaction of free electrons with the electric field of the laser was treated, and the inverse bremsstrahlung process was included.

Our initial configuration was given by a smooth atomic density function, representing a spherically symmetric cluster consisting of 909 neutral xenon atoms. Edges of this sample were smoothed to facilitate computation. The density in the center was comparable to that of the xenon cluster, $\sim 0.0051 / \AA^{3}$. The radius of this cluster was $\sim 25 \AA$. This sample was irradiated with the VUV FEL photons of energies, $E_{\gamma}=12.7 \mathrm{eV}$. For simplicity we have assumed that the photon pulse had a constant intensity, and that it was switched on instantaneously at $t=0 \mathrm{fs}$. The pulse intensity corresponded to an upper estimate of the maximal FEL pulse intensity observed in the experiment [11], $I=10^{14} \mathrm{~W} / \mathrm{cm}^{2}$.

\subsection{Pure Coulomb dynamics inside the irradiated cluster}

In this case we have applied the following simplifying assumptions to the electron and ion/atom dynamics. First, we have expanded the electron density using the angular moment expansion:

$$
\rho^{(e)}(\mathbf{r}, \mathbf{v}, t) \cong \frac{1}{4 \pi}\left(\rho_{0}^{(e)}(r, v, t)+\cos \left(\theta_{v r}\right) \cdot \rho_{1}^{(e)}(r, v, t)\right),
$$

and within this diffusion approximation [25] kept only: (i) its zeroth order (isotropic) component, $\rho_{0}^{(e)}(r, v, t)$, which corresponds to the number of electrons inside a volume element 
$d V=d^{3} r d^{3} v$, and (ii) its first order (transport) component, $\rho_{1}^{(e)}(r, v, t)$, which contributes to the particle flux through the borders of the phase space element. ${ }^{3}$ Radius, $r$, is the distance from the centre of the sample $(r=|\mathbf{r}|)$, and $v$ denotes the magnitude of the electron velocity $(v=|\mathbf{v}|)$. The function $\cos \left(\theta_{v r}\right)$ denotes the cosine of the relative angle between vectors $\mathbf{v}$ and $\mathbf{r}$. The isotropic component of the electron density, $\rho_{0}^{(e)}(r, v, t)$, has to be a positive number, as it describes the number of electrons in an infinitesimal volume element, $d V=d^{3} r d^{3} v$. The transport component of the density, $\rho_{1}^{(e)}(r, v, t)$, can be a positive or a negative number. Positive values of $\rho_{1}^{(e)}$ indicate that there is a collective transport in phase space outwards the sample, the negative ones indicate that there is a collective transport inwards the sample.

The approximation (8) is valid only if the densities, $\rho_{0}^{(e)}(r, v, t)$ and $\rho_{1}^{(e)}(r, v, t)$ fulfill the condition:

$$
\rho_{0}^{(e)}(r, v, t)>>\left|\rho_{1}^{(e)}(r, v, t)\right| .
$$

Within the approximation (8) we neglected the drift component of the electron density which is coupled to the laser field. This component will be treated in the next study case.

Second, as we are only interested in following the electron dynamics within this simplified model, we further assume that the positions of ions are fixed and their velocities remain equal to zero during the evolution:

$$
\rho^{(i)}(\mathbf{r}, \mathbf{v}, t) \cong \frac{1}{4 \pi} \rho_{0}^{(i)}(r, v, t) \cdot \frac{\delta(v)}{v^{2}} .
$$

In the true physical case this assumption is valid only during the first stages of the exposure, as ions are much heavier than electrons (for Xe: $M_{X e} \sim 10^{5} m_{e}$ ). The approximation of a frozen xenon gas (10) implies that $\bar{\rho}^{(i)}(\mathbf{r}, t)=\rho_{0}^{(i)}(r, v=0, t)$.

Within the diffusion approximation (8) Boltzmann equations for the electron density, Eqs. (5), reduce to:

$$
\begin{aligned}
\frac{\partial \rho_{0}^{(e)}(r, v, t)}{\partial t}+ & \frac{v}{3 r^{2}} \cdot \frac{\partial\left(r^{2} \rho_{1}^{(e)}(r, v, t)\right)}{\partial r}-\frac{A\left(r, \rho_{0}^{(i)}, \rho_{0}^{(e)}\right)}{3 v^{2}} \cdot \frac{\partial\left(v^{2} \rho_{1}^{(e)}(r, v, t)\right)}{\partial v}= \\
=\sum_{i=0}^{N_{J}} \rho_{0}^{(i)}(r, 0, t)\{ & j\left(E_{\gamma}\right) \sigma_{\gamma}^{i}\left(E_{\gamma}\right) \frac{\delta\left(v-v_{E}\right)}{v^{2}}+\int_{0}^{\infty} d v_{e} v_{e}^{3} \rho_{0}^{(e)}\left(r, v_{e}, t\right) d \sigma_{t o t}^{i}\left(v_{e} ; v\right) \\
& \left.-v \rho_{0}^{(e)}(r, v, t) \sigma_{t o t}^{i}(v)\right\}
\end{aligned}
$$

\footnotetext{
${ }^{3}$ Within the diffusion approximation the total electron flux in real space through the sphere of radius, $r$, is: $S(r)=\frac{4 \pi}{3} \int_{0}^{\infty} d v v^{3} r^{2} \rho_{1}^{(e)}(r, v, t)$, and the total flux in velocity space through the sphere of radius, $v$, is: $S(v)=-\frac{4 \pi}{3} \int_{0}^{\infty} d r A\left(r, \rho_{0}^{(i)}, \rho_{0}^{(e)}\right) v^{2} r^{2} \rho_{1}^{(e)}(r, v, t)$, where $A\left(r, \rho_{0}^{(i)}, \rho_{0}^{(e)}\right)$ is the radial acceleration (12).
} 


$$
\begin{aligned}
& \frac{\partial \rho_{1}^{(e)}(r, v, t)}{\partial t}+v \cdot \frac{\partial \rho_{0}^{(e)}(r, v, t)}{\partial r}-A\left(r, \rho_{0}^{(i)}, \rho_{0}^{(e)}\right) \cdot \frac{\partial \rho_{0}^{(e)}(r, v, t)}{\partial v}= \\
= & -\sum_{i=0}^{N_{J}} \rho_{0}^{(i)}(r, 0, t) v \rho_{1}^{(e)}(r, v, t) \sigma_{t o t}^{i}(v),
\end{aligned}
$$

where:

$$
\begin{aligned}
A\left(r, \rho_{0}^{(i)}, \rho_{0}^{(e)}\right) & =\frac{e^{2}}{4 \pi \epsilon_{0} m} \cdot \frac{4 \pi}{r^{2}} \cdot \int_{0}^{r} d r^{\prime} r^{\prime 2}\left\{\sum_{i=0}^{N_{J}} i \cdot \rho_{0}^{(i)}\left(r^{\prime}, 0, t\right)-\int_{0}^{\infty} d v v^{2} \rho_{0}^{(e)}\left(r^{\prime}, v, t\right)\right\}, \\
d \sigma_{t o t}^{i}\left(v_{e} ; v\right) & =\sigma_{e c}^{i \rightarrow i}\left(v_{e}\right) \frac{\delta\left(v-v_{e}\right)}{v^{2}}+\frac{2}{v^{2}} \frac{d \sigma_{i c}^{i \rightarrow i+1}\left(v_{e} ; v\right)}{d v} \\
\sigma_{t o t}^{i}(v) & =\sigma_{e c}^{i \rightarrow i}(v)+\sigma_{i c}^{i \rightarrow i+1}(v), \\
\sigma_{\gamma}^{i}\left(E_{\gamma}\right) & \equiv \sigma_{\gamma}^{i \rightarrow i+1}\left(E_{\gamma}\right) .
\end{aligned}
$$

The Coulomb electrostatic force in Eq. (11) has been expanded using the multipole expansion with the accuracy consistent with the accuracy of the diffusion approximation (8). Electron-ion and electron-atom collisions were assumed to be isotropic which is a reasonable approximation at low impact energies of electrons. For the simulation purpose the delta-like photoionization velocity distribution, $\frac{\delta\left(v-v_{E}\right)}{v^{2}}$ in Eq. (11), had to be approximated with a gaussian profile of a non-zero width and a mean value at the photoelectron velocity, $v_{E}$. The normalization constant of this gaussian profile was chosen in order to obtain the correct number of the photoelectrons released.

Inverse bremsstrahlung process or any other heating mechanism were not included within this study case.

As there is no transport of ions within the approximation, Eq. (10), Boltzmann equation for ions (6) reduces to an ordinary rate equation, describing the change of the number of ions due to the photo- and collisional ionizations:

$$
\begin{aligned}
\frac{\partial \rho_{0}^{(i)}(r, 0, t)}{\partial t} & =\rho_{0}^{(i)}(r, 0, t)\left\{j\left(E_{\gamma}\right) \sigma_{\gamma}^{i-1}\left(E_{\gamma}\right)+\int_{0}^{\infty} d v_{e} v_{e}^{3} \rho_{0}^{(e)}\left(r, v_{e}, t\right) \sigma_{i c}^{i-1}\left(v_{e}\right)\right\}- \\
& -\rho_{0}^{(i)}(r, 0, t)\left\{j\left(E_{\gamma}\right) \sigma_{\gamma}^{i}\left(E_{\gamma}\right)+\int_{0}^{\infty} d v_{e} v_{e}^{3} \rho_{0}^{(e)}\left(r, v_{e}, t\right) \sigma_{i c}^{i}\left(v_{e}\right)\right\},
\end{aligned}
$$

where $i=1,2, \ldots, N_{J}$. For atomic densities this equation simplifies to:

$$
\frac{\partial \rho_{0}^{(0)}(r, 0, t)}{\partial t}=-\rho_{0}^{(0)}(r, 0, t)\left\{j\left(E_{\gamma}\right) \sigma_{\gamma}^{0}\left(E_{\gamma}\right)+\int_{0}^{\infty} d v_{e} v_{e}^{3} \rho_{0}^{(e)}\left(r, v_{e}, t\right) \sigma_{i c}^{0}\left(v_{e}\right)\right\}
$$

We have prepared a code dedicated for solving Boltzmann equations for a spherically symmetric atomic cluster located inside a simulation box of a finite size. This code has been based on relevant numerical methods [36-38]. Integrals and partial derivatives in Boltzmann equations were evaluated using the pseudospectral method [37]. Our algorithm has been carefully tested, e.g. the interactions terms were included step-by-step into the code, the 
energy and the particle number were monitored during the evolution. We have proved that our algorithm was conservative so that particle number and energy were conserved with a good accuracy with the Boltzmann equations if the source terms were equal to zero. The accuracy of the time integration has been checked with two independent time-integration methods. Obtaining the predictions for a single case took several hours on the AlphaStation XP1000.

As in this study case the system of electrons and frozen ions reached equilibrium within $10 \mathrm{fs}$ of the exposure, we followed the evolution of the sample up to $50 \mathrm{fs}$ of the exposure. The simulation box had the size: $(0<r<120 \AA) \times(0<v<30 \AA / \mathrm{fs})$, and it was divided into $40 \times 70$ grid points respectively. The simulation box corresponded to a sphere in real space of radius, $r=120 \AA$, and a sphere of radius, $v=30 \AA / \mathrm{fs}$, in velocity space. This box was surrounded by an absorbing wall. Figs. 1-11 show the results.

The quantities obtained after solving Boltzmann equations were: the three-dimensional electron density functions, $\rho_{0}^{(e)}(r, v, t), \rho_{1}^{(e)}(r, v, t)$, and the integrated two-dimensional ion/atom distributions, $\bar{\rho}^{(i)}(\mathbf{r}, t)$, recorded at different times, $t=0, \ldots, 50$ fs. Figs. 1, 2 show an example of the isotropic and the transport component of the electron density in phase space obtained with Boltzmann equations at time, $t=2$ fs. Plotted are the functions: $\tilde{\rho}_{j}^{(e)}(r, v, t)=$ $r^{2} v^{2} \rho_{j}^{(e)}(r, v, t)$, where $j=0,1$. The isotropic component of the electron density function, $\tilde{\rho}_{0}^{(e)}(r, v, t)$, is a positively defined function (Fig. 1). This function is localized in phase space (see the contour plot). In contrast, the transport component of the electron density, $\tilde{\rho}_{1}^{(e)}(r, v, t)$, may take both positive and negative values. In the contour plot of Fig. 2 , the upper part of the contour at $v=4-8 \AA$ ffs with a peak at negative values of $\tilde{\rho}_{1}^{(e)}(r, v, t)$ indicates the inward transport. The lower part of the contour plot at $v=1-3 \AA / \mathrm{fs}$ with a peak at positive values of $\tilde{\rho}_{1}^{(e)}(r, v, t)$ indicates the outward transport.

These three-dimensional plots are not easy to analyze. More transparent information on the evolution of the electron cloud can be obtained from plots of the integrated isotropic and transport density functions, $n$, defined as,

$$
\begin{aligned}
n_{j}(v, t) & \equiv \int \rho_{j}^{(e)}(r, v, t) r^{2} d r \\
n_{j}(r, t) & \equiv \int \rho_{j}^{(e)}(r, v, t) v^{2} d v .
\end{aligned}
$$

The integrated isotropic component, $n_{0}(v, t)$ and $n_{0}(r, t)$ are related to the full integrated densities as, $\bar{\rho}^{(e)}(\mathbf{r}, t)=n_{0}(r, t)$, and, $\bar{\rho}^{(e)}(\mathbf{v}, t)=n_{0}(v, t)$, within the diffusion approximation (8). For ions we have: $n_{0}^{(i)}(r, t) \equiv \rho_{0}^{(i)}(r, 0, t)$. The total number of electrons (ions), $N^{(e, i)}(t)$, can then be obtained after performing the integration of the isotropic component of the density function over $d^{3} r d^{3} v$ :

$$
4 \pi \cdot \int \rho_{0}^{(e, i)}(r, v, t) r^{2} v^{2} d r d v=N^{(e, i)}(t) .
$$


Fig. 3 show the atomic, single ion and double ion density functions. Almost all photoelectrons have been released within the first femtosecond of the exposure. This result is consistent with the photoionization rate estimated at this photon energy, the assumed pulse intensity and the pulse shape. Atomic density significantly decreased within the first femtosecond of the exposure. In contrast, the single ion density at $t=1$ fs followed the shape of the initial atomic distribution at $t=0$ fs. There were no double or highly charged ions observed in the sample, as the electrons released were not energetic enough for further collisional ionizations. Also, photons were assumed to induce single photoionizations of the neutral atoms only.

The dynamics of electrons was strongly non-equilibrium during the first stages of the exposure. Interparticle Coulomb forces were preventing most of the electrons from leaving the ionic sample. Some of the electrons were, however, able to escape. The largest flows of energy and particles have been observed within 5-15 fs of the exposure (Fig. 6d). Within this time weak collective oscillations of the electron cloud around the ion cloud were observed (not shown). After this time electrons thermalized, and their anisotropic transport component became negligible. Fig. 4 shows the rapid progress of the thermalization process for both the isotropic and the transport components of the integrated electron density, $n_{0}(v, t)$ and $n_{1}(v, t)$. The initial free electron density was equal to zero. After the first femtosecond of the exposure the shape of the isotropic electron density, $n_{0}(v, t)$, followed the gaussian profile of the photoelectron velocity distribution, and it broadened with time. Full thermalization was achieved at times $\geq 10$ fs within this test model. We have fitted Maxwell-Boltzmann distribution to the results on the integrated density function, $n_{0}(v, t)$, obtained at $t=20$ fs (Fig. 4): $n_{0}(v, t)=a \cdot \exp \left(-m v^{2} /\left(2 k_{B} T\right)\right)$. The electron temperature was estimated to, $k_{B} T=0.68-0.77 \mathrm{eV}$, which corresponded to the average energy, $\langle E\rangle=3 k_{B} T / 2=$ $1.02-1.15 \mathrm{eV}$. This value agreed well with the average energy estimated with the global parameters at $t=20 \mathrm{fs}$ (Fig. 6a,c), $\langle E\rangle \equiv E_{\text {kinet }} / N_{\text {el }}=1.07 \mathrm{eV}$.

Here we again recall that the energy transferred to the system by a single photon of energy, $E_{\gamma}=12.7 \mathrm{eV}$, was $E_{p h-e l}=1.1 \mathrm{eV}$ instead of $0.6 \mathrm{eV}$, as we had to approximate the delta-like photoionization velocity distribution, $\frac{\delta\left(v-v_{E}\right)}{v^{2}}$ in Eq. (11) with a gaussian profile of a non-zero width.

Fig. 4b shows the time evolution of the transport component of the electron density, $n_{1}(v, t)$, corresponding to the weak plasma oscillations. There is a strong increase of the outward electron transport (in velocity space) within $2 \mathrm{fs}$ of the exposure. Energetic electrons can then leave the simulation box (Fig. 6d). However, at some time point, $t \sim 5$ fs, slower electrons travelling outward are stopped and attracted back by ions. The inward transport start then to dominate. After the thermalization of the electrons is achieved, the 
collective transport (in velocity space) reduces significantly, and the transport component of the electron cloud becomes small.

Spatial evolution of the electron cloud (Fig. 5), described by the integrated densities, $n_{0}(r, t)$ and $n_{1}(r, t)$, is less dynamic than the evolution of the velocity densities, $n_{0}(v, t)$ and $n_{1}(v, t)$. A rapid increase of $n_{0}(r, t)$ is observed only within the first femtosecond of the exposure. After this time, the shape of the isotropic component of the integrated electron density does not change much with time. Weak plasma oscillations are visible, if $r^{2} n_{0}(r, t)$ is plotted (not shown). The magnitude of the spatial transport component of the density function, $n_{1}(r, t)$, is much smaller than its isotropic component, $n_{0}(r, t)$, during the evolution. During the first femtoseconds of the exposure there is a weak spatial transport outward, and the position of the maximum of the transport component propagates towards lower values of $r$ at increasing times. This corresponds to a plasma wave propagating inside the sample. These weak oscillations occur until about $10-20$ fs of the exposure, when they are damped due to the fast progressing thermalization of electrons.

In Fig. 6 we plot also global parameters of the sample as functions of time: (a) the total, kinetic and (b) potential energy of the system, (c) the particle number, and (d) the flows of energy and of the particle numbers recorded at a fixed distance of 10 grid points from the external borders of the simulation box. These flows give a valuable qualitative information about the escape rate of the electrons at different stages of the evolution, which is helpful for estimating the correct size of the simulation box. If the box size would be too small, some electrons of a total negative energy could leave the box during the evolution. This would lead to a strong increase of the potential energy within the system and induce an unrealistic electron dynamics.

Total kinetic energy of the sample and the number of electrons and ions increased rapidly within 2 fs of the exposure. Within and after this time we observed a strong outward flow of electrons. The fastest electrons were able to leave the simulation box. Potential energy slowly increased with time, as more electrons escaped from the simulation box. It was, however, small if compared to the total energy.

\subsection{Extended electron dynamics including drift component of electron density and inverse bremsstrahlung}

Our aim is now to show that this statistical model is also able to predict higher ionization states, as observed in experiments $[11,27]$. Highly charged ions will be created during inelastic collisions of ions with energetic electrons. Other processes can also contribute to the 
formation of highly charged states [15]. Here we concentrate on the collisional ionization that may be a leading process.

Collisional ionization was not possible in the preceding study case 4 . 1. , as we did not include any heating mechanism there, and electrons remained cold during the exposure. Here, we enable electron heating, including the inverse bremsstrahlung process. As we will later see, this process will lead to an efficient heating of electrons, and subsequent collisional ionizations inside the cluster. As we are only interested in following the electron dynamics within this simplified model, we will still keep the assumption of frozen atoms and ions, Eq. (10).

Again, we stress here that within this study case we do not aim to reproduce the experimental results of Refs. [11,27]. This attempt is planned for the forthcoming papers, as it requires detailed adjustments of the present model in order to apply it to a realistic case. Only qualitative comparison of our predictions to the data is possible within this study case.

In order to improve the description of the electromagnetic laser-matter interaction within our cluster, we add a drift component, $\rho_{2}^{(e)}(r, v, t)$, to the diffusion expansion (8):

$$
\rho^{(e)}(\mathbf{r}, \mathbf{v}, t) \cong \frac{1}{4 \pi}\left(\rho_{0}^{(e)}(r, v, t)+\cos \left(\theta_{v r}\right) \cdot \rho_{1}^{(e)}(r, v, t)+\cos \left(\theta_{v \epsilon}\right) \cdot \rho_{2}^{(e)}(r, v, t)\right),
$$

where the function $\cos \left(\theta_{v \epsilon}\right)$ denotes the cosine of the relative angle between vectors: electron velocity, $\mathbf{v}$, and polarization vector of the laser field, $\boldsymbol{\epsilon}$. This component describes electric interaction of the electrons with the field of the laser, and completes the first-order angular moment expansion of $\rho^{(e)}(\mathbf{r}, \mathbf{v}, t)$ up to the terms involving only polar angles. Terms involving azimuthal angles are neglected.

After substituting the ansatz (20) to Eq. (5), we obtain a system of three coupled equations for $\rho_{0}^{(e)}(r, v, t), \rho_{1}^{(e)}(r, v, t)$, and $\rho_{2}^{(e)}(r, v, t)$ (not shown), where we have also included the inverse bremsstrahlung term defined in Eq. (5). We have checked that the term describing the coupling of the inverse bremsstrahlung to the isotropic component of electron density is dominant. We have also proven that at the assumed parameters of the laser field, the argument of Bessel function, $J_{n}^{2}\left(-\frac{e E_{0}}{\hbar \omega^{2}} \boldsymbol{\epsilon}\left(\mathbf{v}_{\mathbf{e}}^{\prime}-\mathbf{v}_{\mathbf{e}}\right)\right)$ is small. Therefore single photon emissions or absorptions will dominate during the inverse bremsstrahlung process.

We have solved the extended equations numerically with our Boltzmann solver. The simulation box had the size: $(0<r<100 \AA) \times(0<v<100 \AA / \mathrm{fs})$, and it was divided into $40 \times 90$ grid points respectively. The radius of the spherically symmetric xenon cluster located inside this simulation box was $\sim 25 \mathrm{~A}$. This cluster was irradiated with an intense pulse of constant intensity, $I=10^{14} \mathrm{~W} / \mathrm{cm}^{2}$. We followed the evolution of this system up to $180 \mathrm{fs}$ of the exposure. At that time the majority of electrons has already left the sample and 
collisional ionization rate saturated. The repulsive Coulomb forces within the sample were so large that Coulomb explosion of ions should have already started. This was, however, not possible within the approximation of frozen ions, and we stopped the simulation at entering this unphysical regime.

As in the previous study case, we followed the evolution of irradiated cluster, recording electron and ion densities at different times of the exposure (not shown). With these observables we estimated global parameters of the system as functions of time (Fig. 7): (a) total, kinetic and potential energy, (b) electron temperature, (c) total number of electrons, atoms and ions, (d) energy and particle flows. In Fig. 8 we also plotted the total numbers of electrons and ions of different charges recorded as a function of time.

Including the drift component (20) into the Boltzmann equations lead to fast collective oscillations of the electron cloud. Such plasma oscillations are expected to appear at intense laser fields, when the strong electric field of the laser is sufficient to drive electron cloud forth and back in the direction of the field polarization. Fig. 9 shows the oscillations of kinetic energy and total energy of the electrons due to this effect.

In our extended model electrons gained energy via the inverse bremsstrahlung process. This effect is reflected in the shapes of the kinetic energy curve and of the temperature curve (Figs. 7a,b). The total kinetic energy of electrons and their temperature increased with time until $\sim 160$ fs of the exposure (Fig. 7a). After this time most of the electrons were energetic enough to leave the simulation box (Fig. 7c,d), and the total kinetic energy of electrons within the box decreased. However, the temperature of electrons within the simulation box still grew for some time as the remaining electrons still gained energy from the laser field with the inverse bremsstrahlung process.

Two phases of fast electron escape occurred at about $10-30$ fs and after $160 \mathrm{fs}$ of the exposure. These phases were reflected in energy flows recorded during the exposure (Fig. $7 d$ ). The first escape phase occurred before highly charged ions (+2 and higher) were created within the sample (Fig. 8a). Some of electrons gained the amount of energy sufficient to leave the sample and the simulation box. This escape phase ended, when the attracting Coulomb force within the sample increased. It then kept most of the electrons inside the sample until the second escape phase started at about 160 fs. Within this phase a majority of electrons left the simulation box. If the ions were not frozen, this should have lead to a fast Coulomb explosion of remaining ions.

Between these two escape phases highly charged ions have been created in the subsequent ionization processes: $X e^{+q} \rightarrow X e^{+q+1}$, where $q=0, \ldots, 6$. Doubly charged ions were observed after $5-6$ fs of the exposure. Triply charged ions were observed after $30-40$ 
of the exposure. Ions of charges +4 up to +7 were created later in the exposure, starting at $70(\mathrm{Xe}+4)$ and $120 \mathrm{fs}(\mathrm{Xe}+7)$ of the exposure.

At the end of the exposure ( $t=180 \mathrm{fs}$ ) there were no neutral atoms left within the cluster. Contributions of ions of specific charges to the total number of ions were following: Xe +1 $(\sim 10 \%), \mathrm{Xe}+2(\sim 7 \%), \mathrm{Xe}+3(\sim 11 \%), \mathrm{Xe}+4(\sim 7 \%), \mathrm{Xe}+5(\sim 10 \%), \mathrm{Xe}+6(\sim 16 \%)$ and $\mathrm{Xe}+7(\sim 39 \%)$. The contribution of $\mathrm{Xe}+7$ ions was the largest one.

\section{Summary and conclusions.}

We have formulated general Boltzmann equations describing the evolution of a non-uniform sample irradiated with VUV FEL photons. We have solved these equations numerically with a dedicated algorithm. Two study cases of a simplified electron dynamics inside a spherically symmetric xenon cluster were considered. In case when the laser-matter interaction was restricted to the photoionization effect, results obtained with Boltzmann equations gave a comprehensive description of the evolution of electron cloud during its non-equilibrium (before thermalization) and equilibrium stages (after thermalization). At photon energies, $E_{\gamma}=12.7 \mathrm{eV}$, thermalization of electrons was observed after $10 \mathrm{fs}$ of the exposure within this system. This thermalization was an effect of long-range Coulomb forces, and not of the interparticle collisions, as the energy transfers in the non-ionizing electron collisions were not allowed within this model.

During the exposure almost all photoelectrons were confined inside the ion cluster by the Coulomb internal field. Only a few photoelectrons were energetic enough to escape. Therefore the electrostatic energy of the sample was too low to accelerate remaining electrons and initiate further collisional ionizations. These results confirm that efficient mechanisms of energy pumping are necessary for the creation of highly charged ions in the sample irradiated with VUV photons.

In the second study case we extended the description of the laser-matter interaction including the drift component of electron density. This drift component induced fast collective oscillations of the electron cloud in the intense electric field of the laser.

In this case the inverse bremsstrahlung process was also introduced as a mechanism of energy pumping. We then observed higher ionization states created within the irradiated cluster after about 6 fs $(\mathrm{Xe}+2), 30 \mathrm{fs}(\mathrm{Xe}+3), 70 \mathrm{fs}(\mathrm{Xe}+4), 90 \mathrm{fs}(\mathrm{Xe}+5), 100 \mathrm{fs}(\mathrm{Xe}+6)$ and $120 \mathrm{fs}(\mathrm{Xe}+7)$ of the exposure. These states were created during inelastic collisions of energetic electrons with ions. Simulation ended up with the majority of Xe +7 ions $(\sim 40 \%)$ 
within the sample.

Two phases of fast electron escape were observed during the exposure. After the second escape phase majority of the electron has left the sample, and at this time the Coulomb explosion of unscreened ions should have started. This was not possible, as the ions were kept frozen within this model.

The results obtained with the extended model are promising. We observe a qualitative agreement between the charge densities and the average energy absorbed per atom estimated with this primary model and the experimental data. In the first experiments [11] at a power density in the range $10^{13}-10^{14} \mathrm{~W} / \mathrm{cm}^{2}$ charge states up to $8+$ where observed. The kinetic energy of the ions varies between $100 \mathrm{eV}$ and $2500 \mathrm{eV}$, depending on the charge state. These numbers are in the same range as the values predicted in the present work. However, at this stage we can make only qualitative comparison of our predictions to the data. The model developed here needs further improvements of its physical assumptions in order to be applied to a realistic case. Effects of plasma screening on atomic energy levels and on photo- and collisional ionization cross sections have to be treated $[9,12]$. So far potentials used in equations were unscreened, and cross sections (also inverse bremstrahlung cross sections) were also obtained with those unscreened potentials. Including the screening effects within this non-uniform sample will significantly affect the ionization dynamics. Within the improved model also the realistic pulse shape, correct cluster size and density should be implemented. Ions should not be kept frozen during the evolution. Important mechanisms of thermalization: recoil effects and short-range electron-electron interactions, need to be treated in this improved model. Possible influence of recombination and of other many body processes on the sample dynamics requires dedicated analysis.

However, we do not expect that including further interactions into these equations will lead to more numerical complicacies. As the main nonlinearity and stability problems have been successfully treated in the primary algorithm, and this algorithm correctly followed the dynamics of the sample in our study cases, we expect that it can easily be extended for a more advanced model.

At this point we have also to discuss the applicability of the classical approximation for describing the evolution of FEL irradiated samples (see also [39]). We stress here that our final aim is to obtain a description of radiation damage at short wavelenghts of photon radiation (soft and hard X-rays), when plasma electrons plasma are hot, and their treatment with classical Boltzmann equations therefore justified. When we try to apply the classical description to a sample irradiated with VUV FEL photons of $\lambda \sim 100 \mathrm{~nm}\left(E_{\gamma}=12.7\right.$ $\mathrm{eV}$ ), and estimate the degeneracy parameter $\Upsilon=E_{\text {Fermi }} / k_{B} T$ for electrons, it is $\sim 1$ for electron plasma of temperature, $T \sim 1 \mathrm{eV}$ and density $n_{e} \sim 10^{22} \mathrm{~cm}^{-3}$, and it is $\sim 20$ for the 
plasma of the same temperature and density $n_{e} \sim 10^{24} \mathrm{~cm}^{-3}$ These values are much above the classical regime, $\Upsilon \ll 1$.

However, the evolution of an irradiated sample is a non-equilibrium process, and if the energy gain by electrons and electron escape rate from the sample were fast enough, the system could enter the classical regime very early in the exposure. Classical description could then still be applicable. This scenario is probable, according to the results obtained with our second study case. Therefore, in the realistic case it will be necessary to monitor the degeneracy parameters during the evolution of the sample. Their value will justify the validity of the classical approximation.

We believe that despite those limitations the method proposed here offers a unique possibility of studying the complex dynamics of large spatially non-uniform samples, irradiated with the FEL pulses. Whereas in real experiments the sample is exposed to several processes contributing simultaneously to the radiation damage, the Boltzmann simulation tool enables one to include specific interactions only. In this way the influence of different ionization mechanisms on the overall dynamics of the sample can conveniently be tested. Also, accurate time characteristics of damage processes can easily be obtained.

To sum up, Boltzmann approach is a first principle model which can follow non-equilibrium classical processes in phase space. Single particle densities evolved with Boltzmann equations include the full information on particle positions and velocites, and not only on their collective components. Average observables obtained with the Boltzman solver are not biased with statistical errors. However, the information on the three and higher order correlations is not included within Boltzmann equations. Including the effects of many body correlations into these classical equations is generally not possible, only in a few cases and under simplifying assumptions, e.g. by applying the Fokker-Planck equation in case of longrange Coulomb forces. The other serious disadvantage of the Boltzmann approach is its numerical complexity which requires an application of advanced numerical methods.

Computational costs within Boltzmann approach do not scale with the number of atoms within a sample, as in the MC method. Therefore, a Boltzmann solver is usually much more efficient for larger samples of a regular structure than a Monte Carlo code. This does not apply for samples of a complex or irregular structure. As these samples cannot be accurately represented by a smooth density function, Boltzmann equations can only give a crude estimate of their damage dynamics. Improving the accuracy within the Boltzmann approach is possible only by extending the number of grid points used to represent the sample. This may lead to very long computational times when a large number of grid points was applied.

To sum up, we have demonstrated that the Boltzmann equations are a useful method to 
follow the radiation damage of non-uniform samples irradiated with the FEL photons. We believe that these equations may soon become a standard tool for investigating the complex dynamics of irradiated samples.

\section{Acknowledgements}

Beata Ziaja is grateful to T. Chmaj, J. Feldhaus, S. Hau-Riege, L. Motyka, R. A. London and B. Sonntag for illuminating discussions. She thanks G. Ingelman for providing an access to the computing units of the high energy physics group at the Uppsala University, and I. Koersner for computer support. This research was supported in part by the Polish Committee for Scientific Research with grant No. 2 P03B 02724. Beata Ziaja is a fellow of the Alexander von Humboldt Foundation.

\section{References}

[1] LCLS: The First Experiments. SSRL, SLAC, Stanford, USA, 2000.

[2] TESLA, the Superconducting Electron-Positron Linear Collider with an integrated Xray Laser Laboratory. Technical Design Report., DESY, ISBN 3-935702-00-0, 5:150$168,2001$.

[3] R. Neutze, R. Wouts, D. van der Spoel, E. Weckert, and J. Hajdu. Nature, 406:752-757, 2000.

[4] Z. Jurek, G. Faigel, and M. Tegze. Eur. Phys. D, 29:217, 2004.

[5] S. P. Hau-Riege, R. A. London, and A. Szöke. Phys. Rev. E, 69:051906, 2004.

[6] T. Ditmire et al. Physical Review A, 53:3379, 1996.

[7] I. Last and J. Jortner. Physical Review A, 60:2215, 1999.

[8] V. P. Krainov and M. B. Smirnov. Phys. Rep., 370:237, 2002.

[9] R. Santra and C. H. Greene. Phys. Rev. Lett., 91:233401, 2003.

[10] U. Saalmann and J.-M. Rost. Phys. Rev. Lett., 89:143401, 2002.

[11] H. Wabnitz et al. Nature, 420:482, 2002. 
[12] C. Siedschlag and J.M. Rost. Phys. Rev. Lett., 93:043402, 2004.

[13] D. Bauer. J. Phys. B, 37:3085, 2004.

[14] C. Jungreuthmayer, L. Ramunno, J. Zanghellini, and T. Brabec. J. Phys. B, 38:3029, 2005.

[15] C. Siedschlag U. Saalmann and J. M. Rost. J. Phys. B, 39:R39, 2006.

[16] A. Heidenreich, I. Last, and J. Jortner. Eur. Phys. J. D, 35:567, 2005.

[17] I. P. Sharkofsky, T. W. Johnston, and M. P. Bachynski. The particle kinetics of plasmas. Addison Wesley Publishing Company, Inc., 1966.

[18] S. Chapman and T. G. Cowling. The mathematical theory of non-uniform gases. Cambridge Univerisy Press, 1970.

[19] V.V. Aristov. Direct methods for solving the Boltzmann equation and the study of nonequilibrium flows. Fluid Mechanics and its Applications; Kluwer Academic Publishers, 60, 2001.

[20] J. M. Buick. Lattice Boltzmann methods in interfacial wave modelling. Doctoral Thesis, University of Edinburgh, page 9, 1997.

[21] W. L. Kruer. The physics of laser plasma interactions. Addison Wesley Publishing Company, Inc., 1988.

[22] W. L. Morgan and B. M. Penetrante. Comp. Phys. Comm., 58:127, 1990.

[23] R. W. Lee. private communication.

[24] D. Loffhagen and R. Winkler. J. Phys. D, 34:1355, 2001.

[25] J. A. Pons et al. Astrophysical J., 513:780, 1999.

[26] A. Mezzacappa et al. Phys. Rev. Lett., 86:1935, 2001.

[27] T. Moeller et al. private communication, 2006.

[28] G. J. Pert. J. Phys. B, 29:1135, 1996.

[29] A. Brantov et al. Physics of Plasmas, 10:3385, 2003.

[30] N. M. Kroll and K. M. Watson. Phys. Rev. A, 8:804, 1973. 
[31] H. Date, Y. Ishimaru, and M. Shimozuma. Nucl. Instr. and Meth. in Phys. Res. B, 207:373, 2003.

[32] H. Tawara and T. Kato. At. Data and Nucl. Data Tabl., 36:168, 1987.

[33] W. F. Chan et al. Phys. Rev. A, 46:149, 1992.

[34] V.P. Shevelko and H. Tawara. J. Phys. B, 28:L589, 1995.

[35] C. Belenger et al. J. Phys. B, 30:2667, 1997.

[36] W. H. Press et al. Numerical Recipes in Fortran 77. Cambridge University Press, 1, 2003.

[37] J. P. Boyd. Chebyshev and Fourier Spectral Methods. Dover Publications Inc., 2000.

[38] D. Gottlieb and S. A. Orszag. Numerical Analysis of Spectral Methods: Theory and Applications. CBMS Conference Series in Applied Mathematics, SIAM, 26, 1977.

[39] I. Last and J. Jortner. Phys. Rev. A, 73:013202, 2006. 


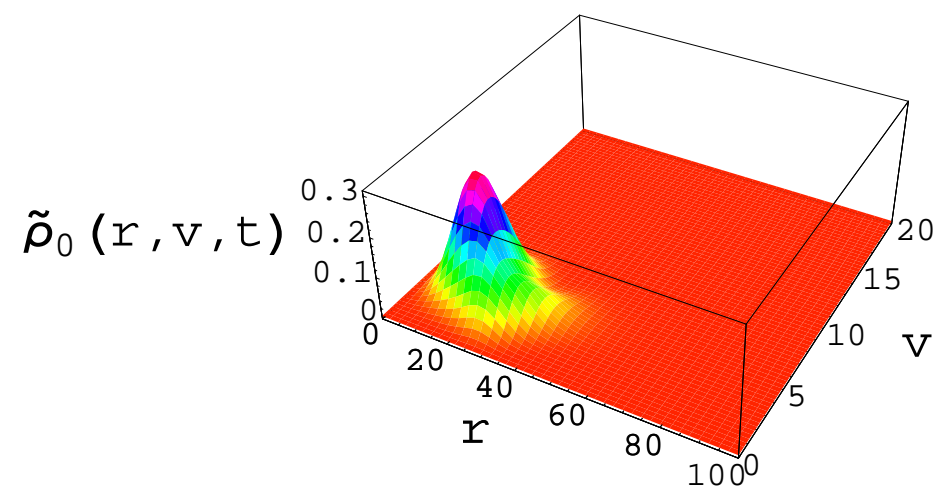

a)

b)

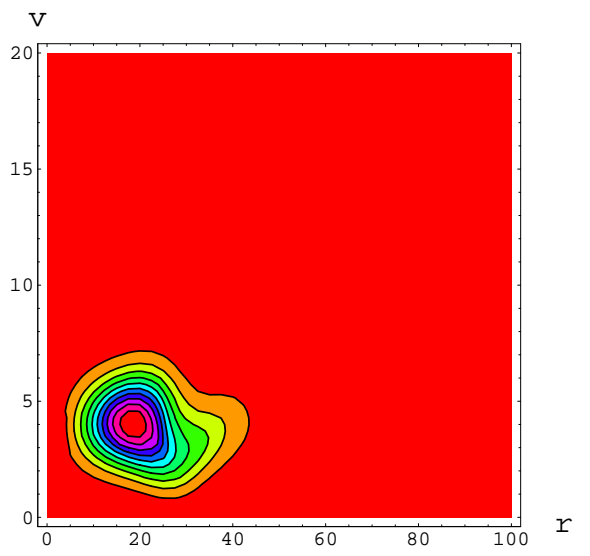

Figure 1: Isotropic component of the electron density in phase-space, $\tilde{\rho}_{0}(r, v, t)=$ $r^{2} v^{2} \rho_{0}(r, v, t)$, recorded at time, $t=2$ fs: a) three-dimensional view and $\mathrm{b}$ ) contour plot. This density was obtained in case of the irradiation with the VUV FEL photons of energies, $E_{\gamma}=12.7 \mathrm{eV}$. Coulomb interactions between charged particles were included. Initial density of free electrons at $t=0$ fs was equal to 0 . The ranges of axes correspond to the size of the simulation box. 


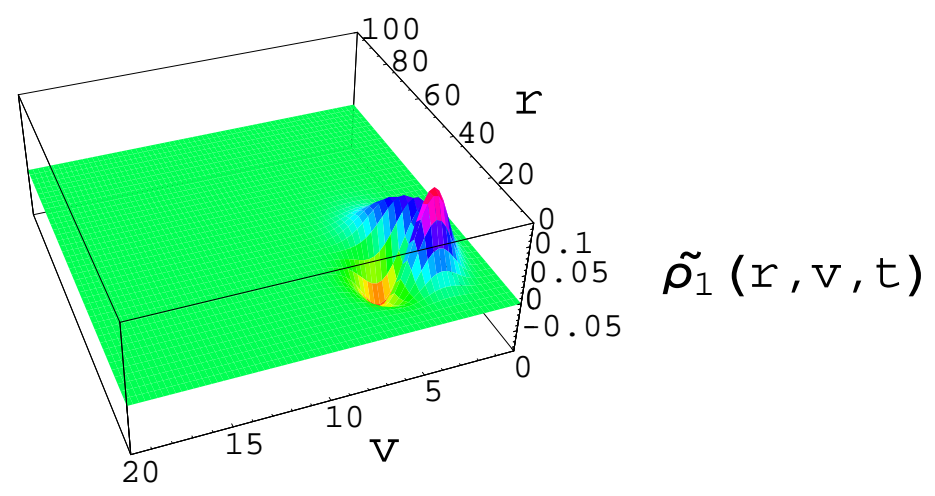

a)

b)

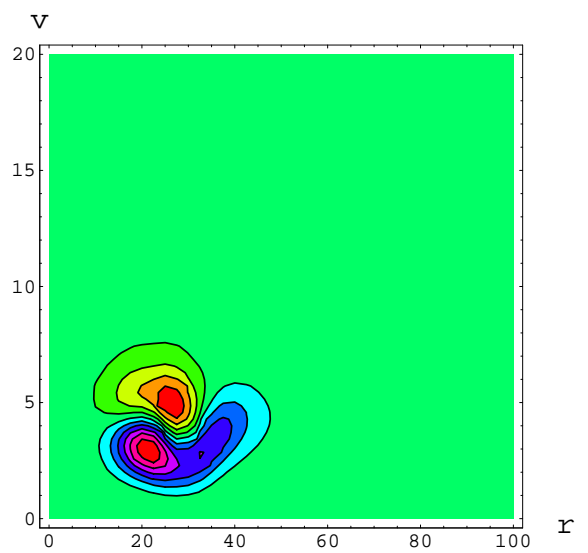

Figure 2: Transport component of the electron density in phase-space, $\tilde{\rho}_{1}(r, v, t)=$ $r^{2} v^{2} \rho_{1}(r, v, t)$, recorded at time, $t=2 \mathrm{fs}$ : a) three-dimensional view and $\mathrm{b}$ ) contour plot. This density was obtained in case of the irradiation with the VUV FEL photons of energies, $E_{\gamma}=12.7 \mathrm{eV}$. Coulomb interactions between charged particles were included. Initial density of free electrons at $t=0$ fs was equal to 0 . The ranges of axes correspond to the size of the simulation box. 

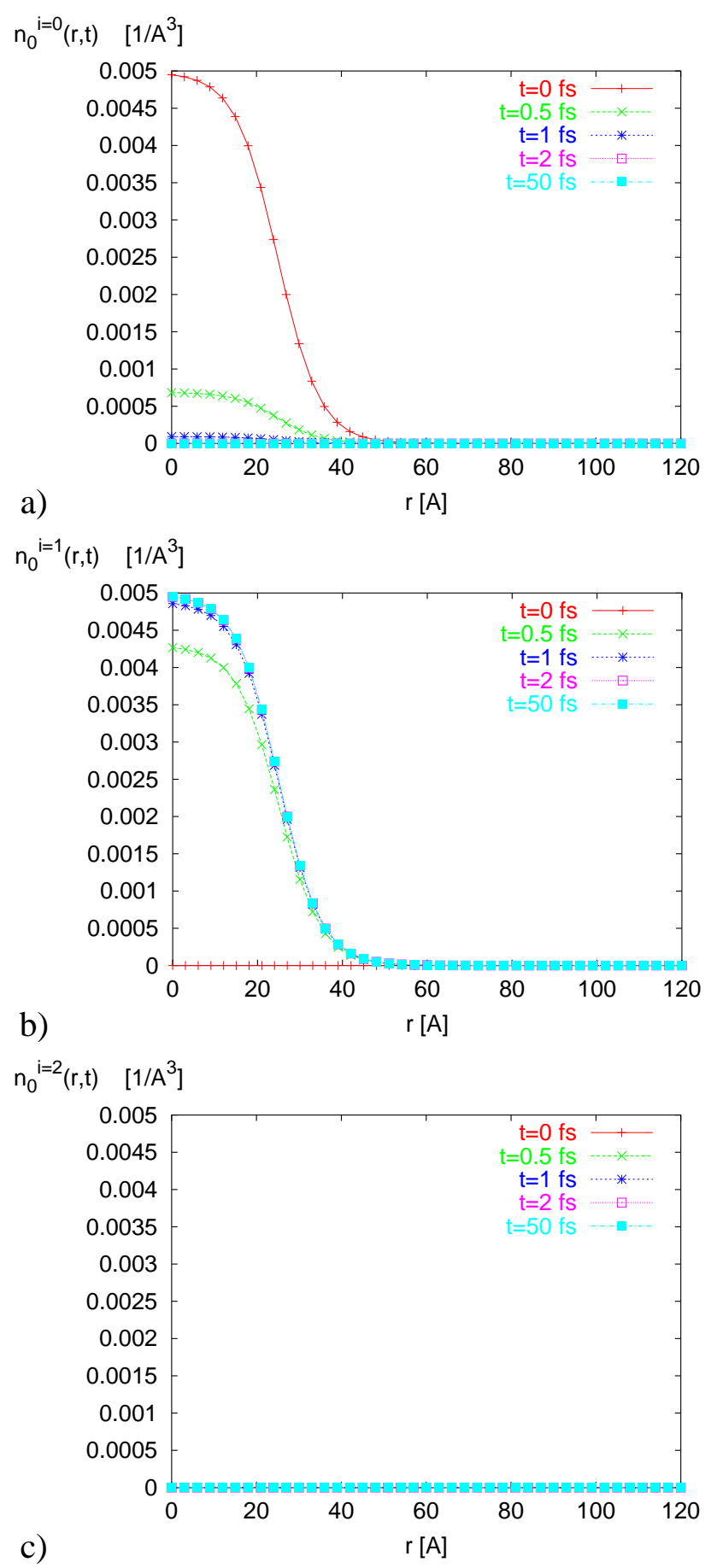

Figure 3: Integrated atom and ion densities, $n_{0}^{i=0,1,2}(r, t)$ : a) atomic density, b) single ion density, c) double ion density, recorded at times, $t=0, \ldots, 50 \mathrm{fs}$. These densities were obtained in case of the irradiation with the VUV FEL photons of energies, $E_{\gamma}=12.7 \mathrm{eV}$. Coulomb interactions between charged particles were included. 


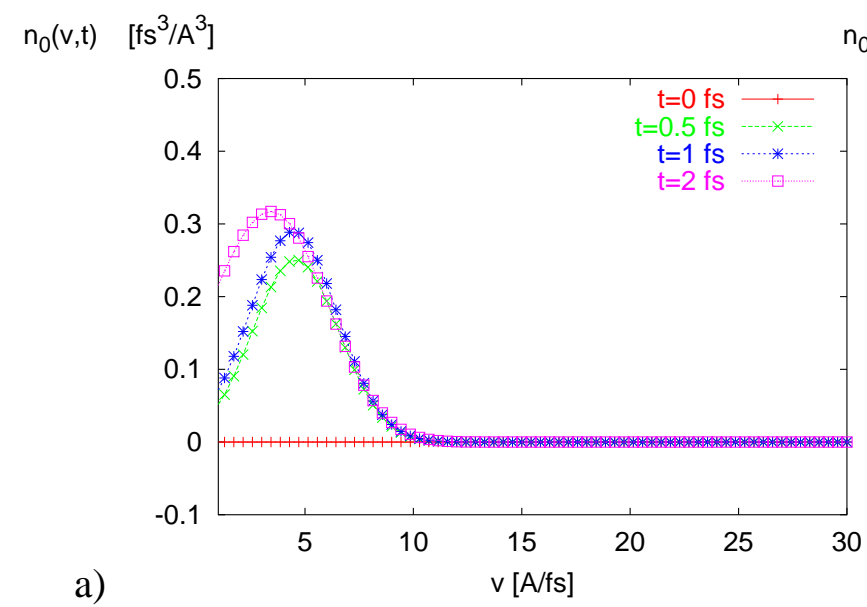

$\mathrm{n}_{0}(\mathrm{v}, \mathrm{t}) \quad\left[\mathrm{fs}^{3} / \mathrm{A}^{3}\right]$

$n_{1}(v, t) \quad\left[\mathrm{fs}^{3} / \mathrm{A}^{3}\right]$

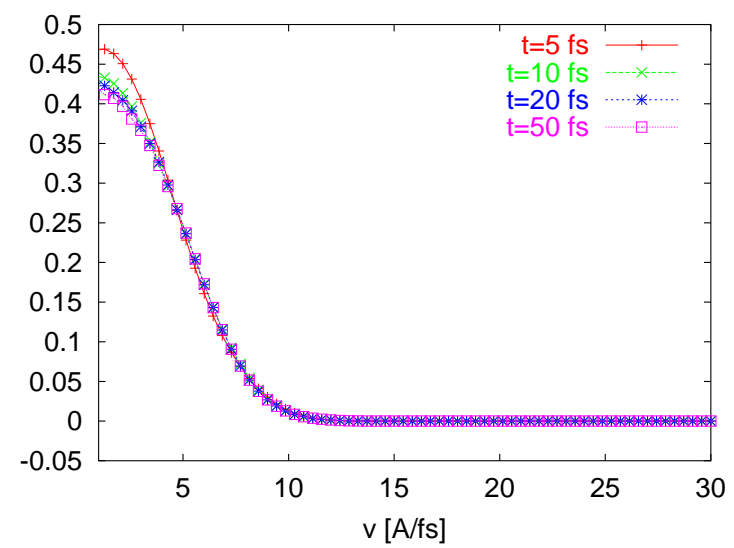

$\mathrm{n}_{1}(\mathrm{v}, \mathrm{t}) \quad\left[\mathrm{fs}^{3} / \mathrm{A}^{3}\right]$
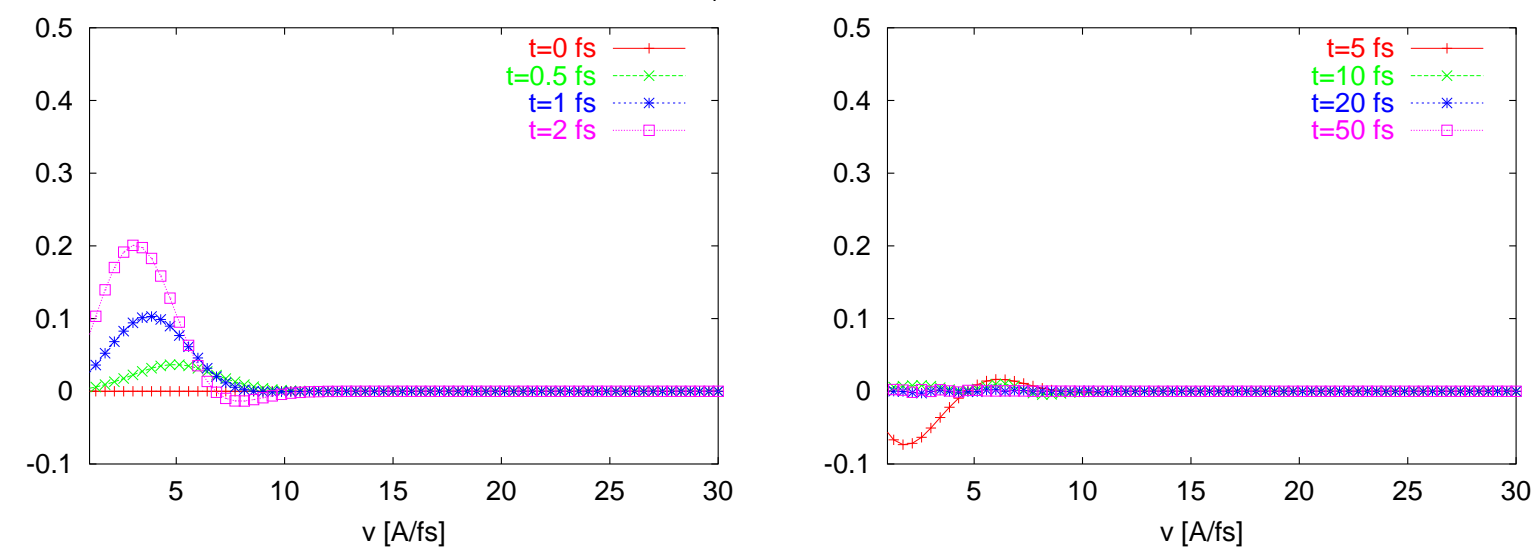

Figure 4: Integrated electron density: a) isotropic component, $n_{0}(v, t)$, b) transport component, $n_{1}(v, t)$, recorded at different times, $t=0, \ldots, 50$ fs. These densities were obtained in case of the irradiation with the VUV FEL photons of energies, $E_{\gamma}=12.7 \mathrm{eV}$. Coulomb interactions between charged particles were included. 

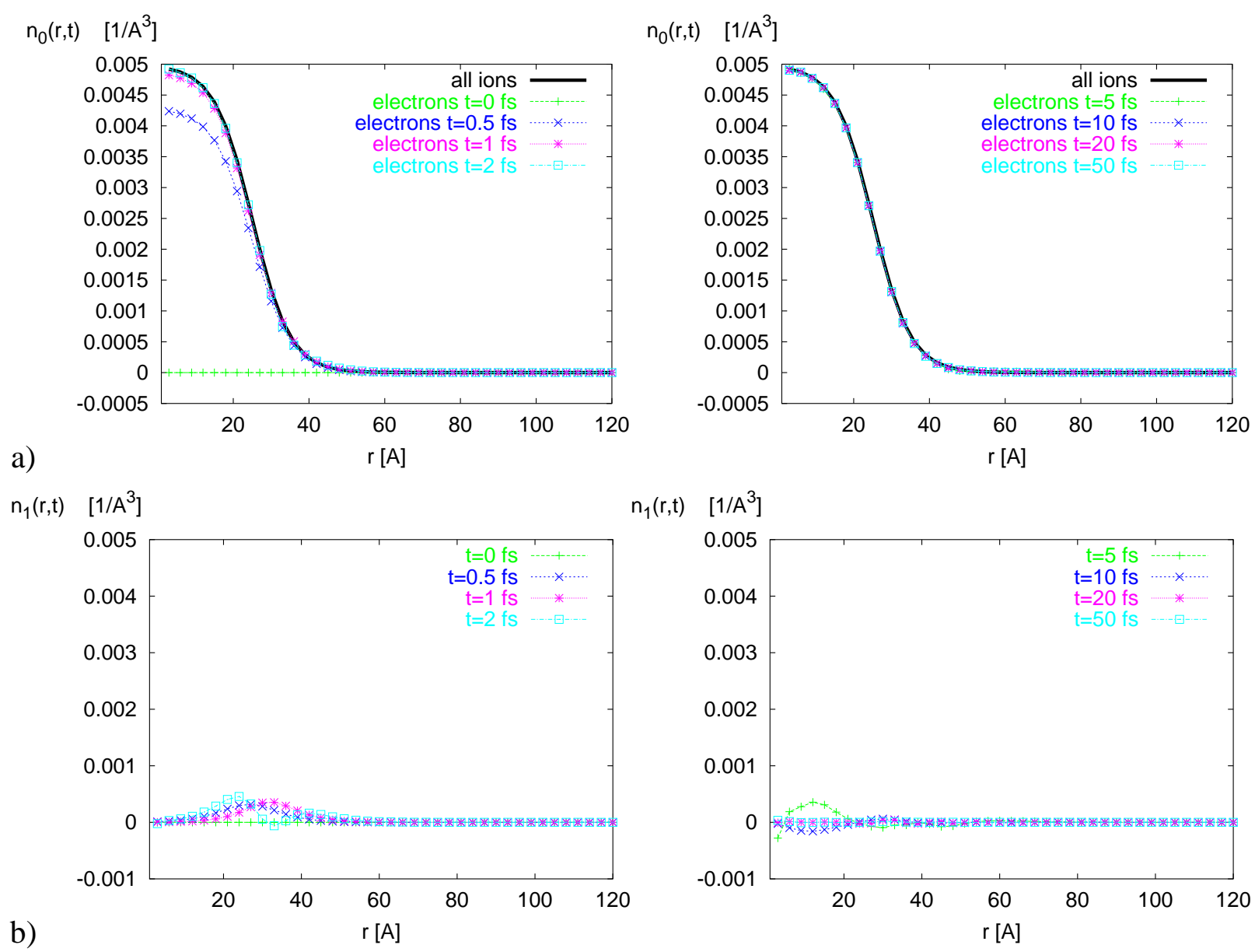

Figure 5: Integrated electron density: a) isotropic component, $n_{0}(r, t)$, b) transport component, $n_{1}(r, t)$, recorded at different times, $t=0, \ldots, 50$ fs. These densities were obtained in case of the irradiation with the VUV FEL photons of energies, $E_{\gamma}=12.7 \mathrm{eV}$. Coulomb interactions between charged particles were included. 

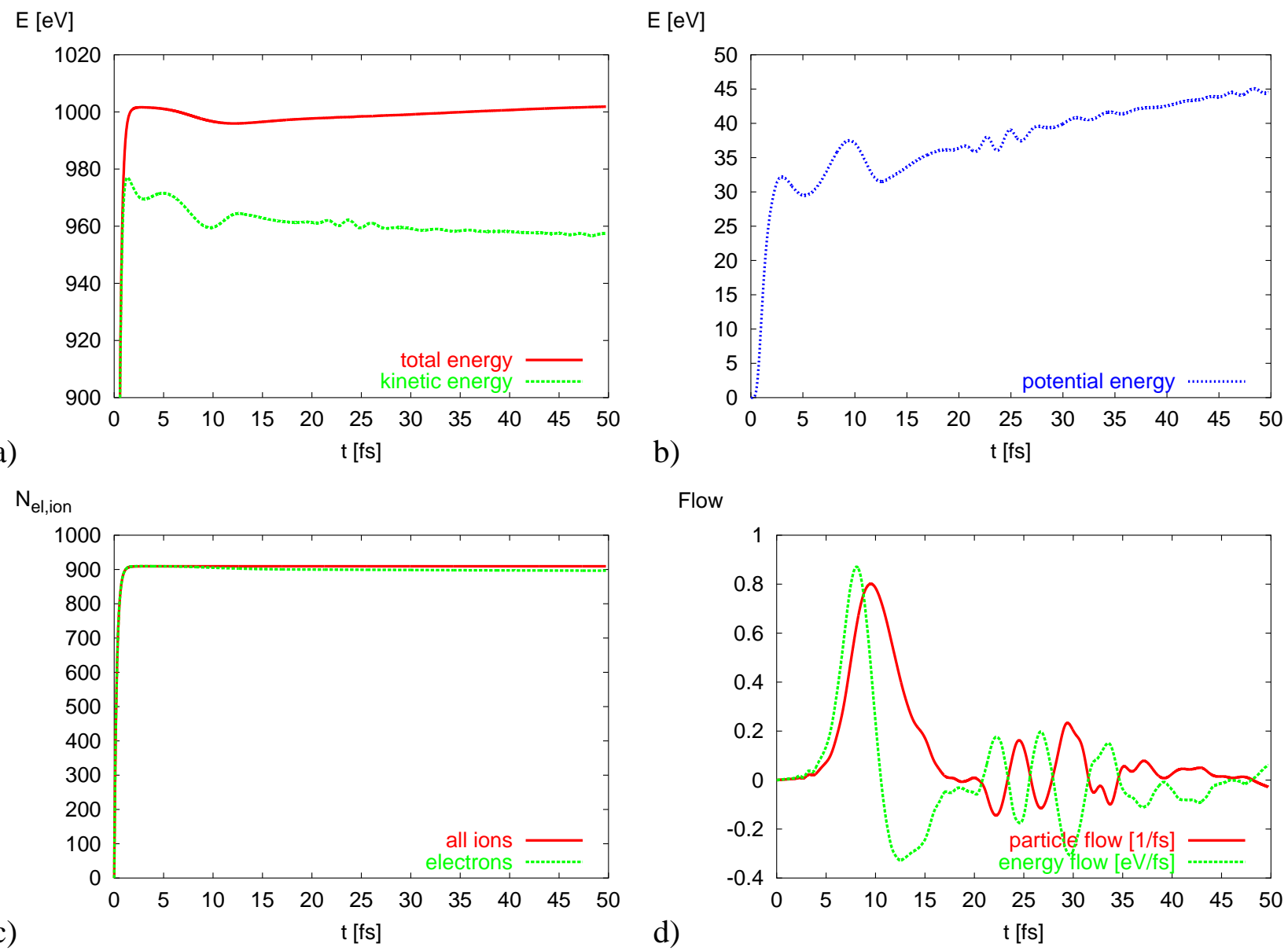

Flow

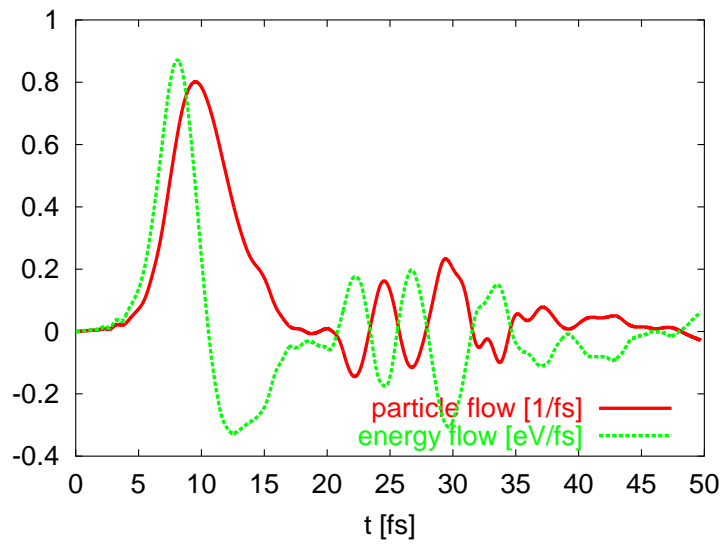

Figure 6: Global parameters of the irradiated sample as functions of time: a) total energy, b) potential energy, c) number of electrons and singly charged ions in the sample, d) flows of energy and particles measured at the distance of 10 grid points from the external borders of the simulation box. These parameters were obtained in case of the irradiation with the VUV FEL photons of energies, $E_{\gamma}=12.7 \mathrm{eV}$. Coulomb interactions between charged particles were included. 

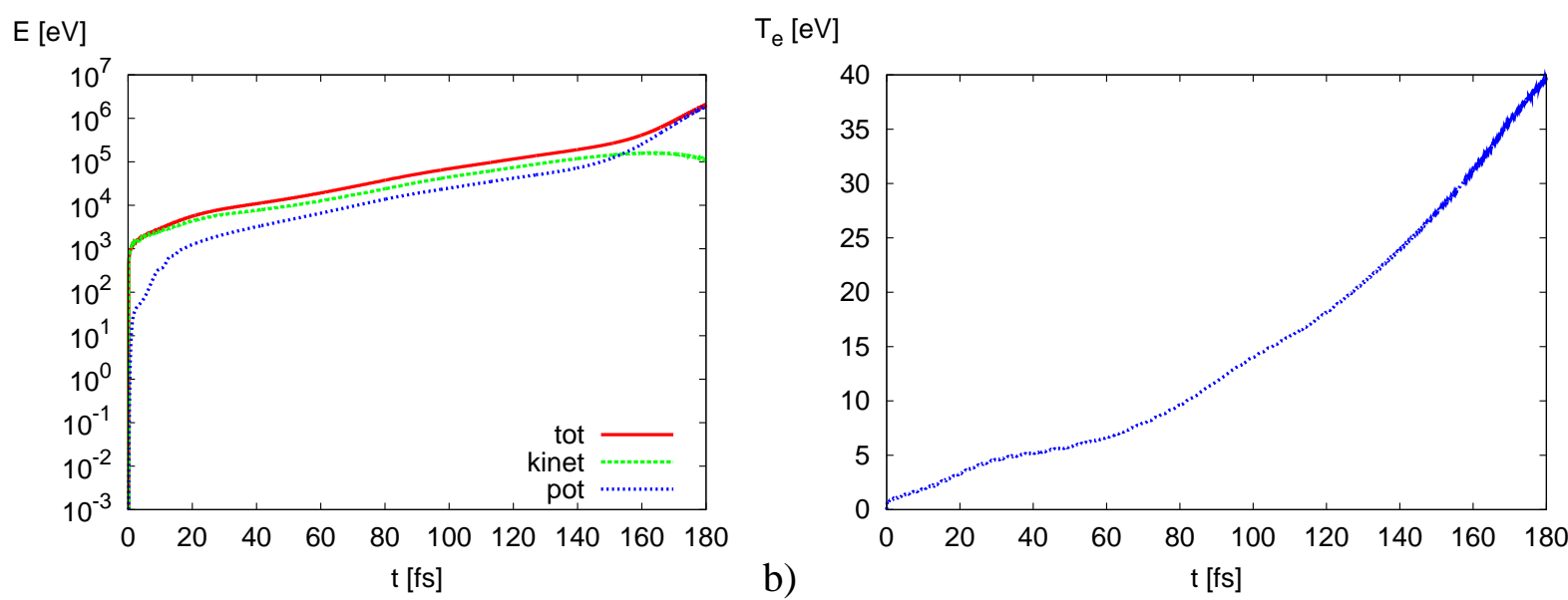

a)

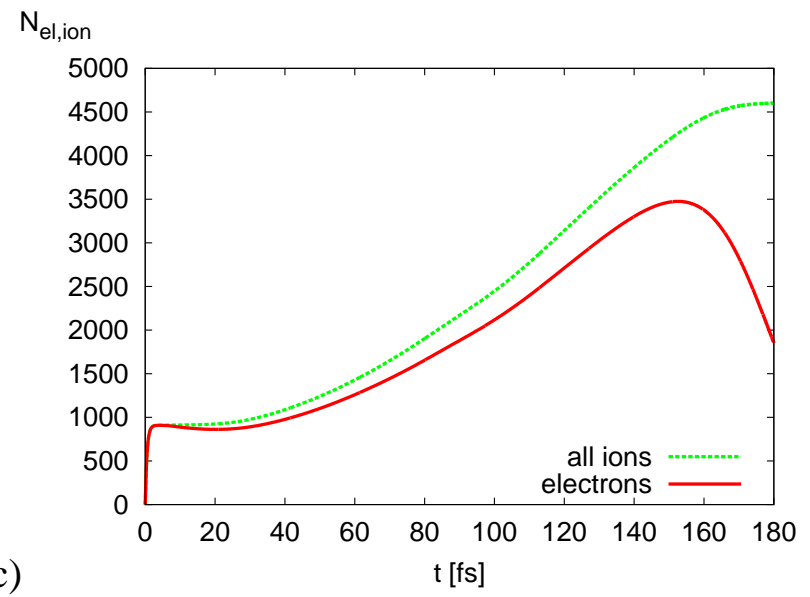

Flow

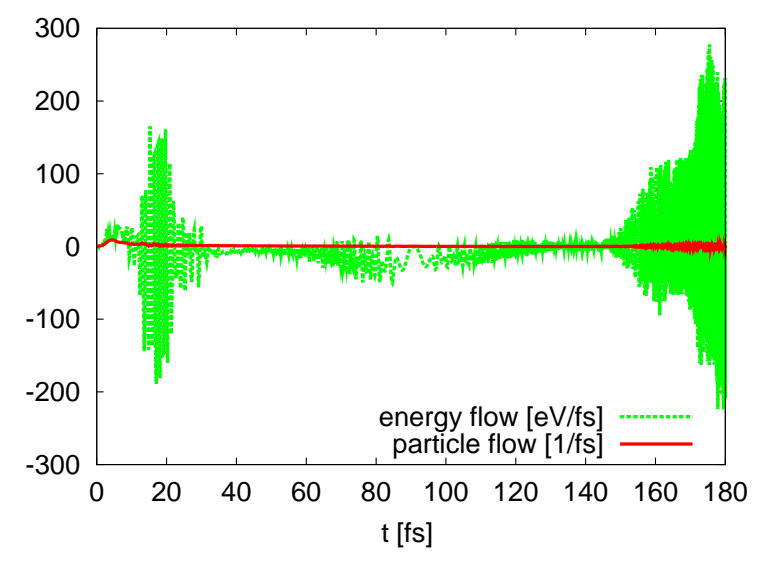

Figure 7: Global parameters of the irradiated sample as function of time: a) total, kinetic and potential energy, b) electron temperature, c) number of electrons and gross-number of ions, $N_{i o n}=\sum_{i} i \cdot N_{i}$, where $N_{i}$ is the number of ions of charge $i, \mathrm{~d}$ ) flows of energy and particles measured at the distance of 16 grid points from the external borders of the simulation box. These parameters were obtained in case of the irradiation with the VUV FEL photons of energies, $E_{\gamma}=12.7 \mathrm{eV}$, in the extended model, where both the inverse bremsstrahlung process and drift component of the electron density were included. 
$\mathrm{N}_{\mathrm{el}, \mathrm{ion}}$

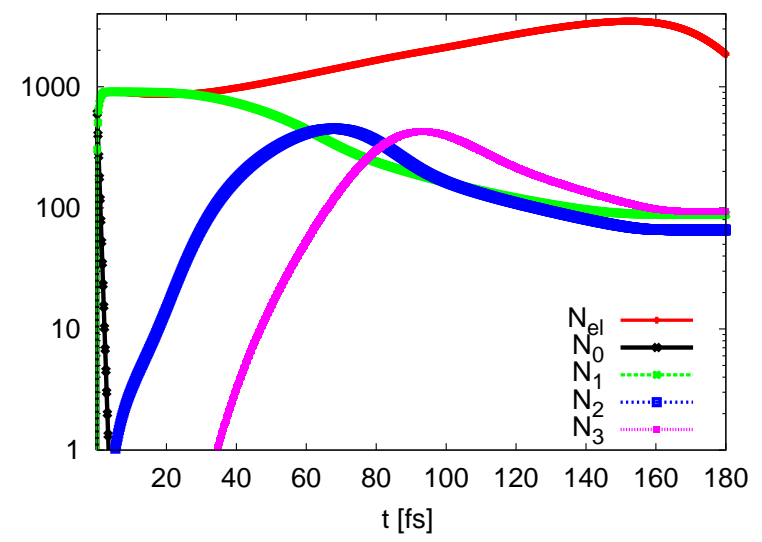

$\mathrm{N}_{\mathrm{el}, \text { ion }}$

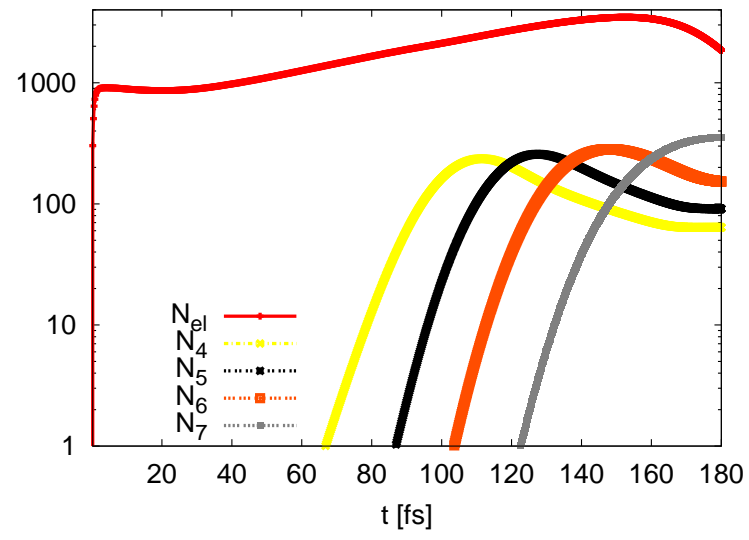

Figure 8: Number of electrons and ions created within the sample during the exposure to VUV FEL photons of energies, $E_{\gamma}=12.7 \mathrm{eV}$ : a) electrons and ions up to +3 , b) electrons and ions from +4 up to +7 . Those results were obtained with the extended model, where both the inverse bremsstrahlung process and drift component of the electron density were included.

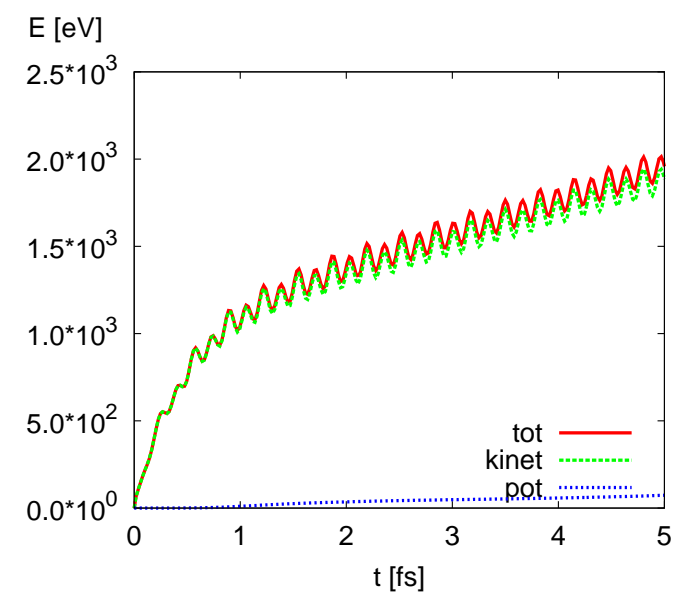

Figure 9: Total, kinetic and potential energy of electrons within the simulation box within first femtoseconds of the exposure. Characteristic plasma oscillations are reflected by kinetic energy curve. Those results were obtained with the extended model, where both inverse bremsstrahlung process and drift component of the electron density were included. 